\title{
PATTERNS IN RANDOM PERMUTATIONS AVOIDING THE PATTERN 132
}

\author{
SVANTE JANSON
}

\begin{abstract}
We consider a random permutation drawn from the set of 132-avoiding permutations of length $n$ and show that the number of occurrences of another pattern $\sigma$ has a limit distribution, after scaling by $n^{\lambda(\sigma) / 2}$ where $\lambda(\sigma)$ is the length of $\sigma$ plus the number of descents. The limit is not normal, and can be expressed as a functional of a Brownian excursion. Moments can be found by recursion.
\end{abstract}

\section{INTRODUCTION}

We say that two sequences (of the same length) $x_{1} \cdots x_{k}$ and $y_{1} \cdots y_{k}$ of real numbers have the same order if $x_{i}<x_{j} \Longleftrightarrow y_{i}<y_{j}$ for all $i, j \in[k]$.

Let $\mathfrak{S}_{n}$ be the set of permutations of $[n]:=\{1, \ldots, n\}$. If $\sigma=\sigma_{1} \cdots \sigma_{k} \in$ $\mathfrak{S}_{k}$ and $\pi=\pi_{1} \cdots \pi_{n} \in \mathfrak{S}_{n}$, then an occurrence of $\sigma$ in $\pi$ is a subsequence $\pi_{i_{1}} \cdots \pi_{i_{k}}$, with $1 \leqslant i_{1}<\cdots<i_{k} \leqslant n$, that has the same order as $\sigma$. We let $n_{\sigma}(\pi)$ be the number of occurrences of $\sigma$ in $\pi$, and note that

$$
\sum_{\sigma \in \mathfrak{S}_{k}} n_{\sigma}(\pi)=\left(\begin{array}{l}
n \\
k
\end{array}\right),
$$

for every $\pi \in \mathfrak{S}_{n}$.

We say that $\pi$ avoids $\sigma$ if $n_{\sigma}(\pi)=0$; otherwise, $\pi$ contains $\sigma$. Let

$$
\mathfrak{S}_{n}(\sigma):=\left\{\pi \in \mathfrak{S}_{n}: n_{\sigma}(\pi)=0\right\},
$$

the set of permutations of length $n$ that avoid $\sigma$. We also let $\mathfrak{S}_{*}(\sigma):=$ $\bigcup_{n=1}^{\infty} \mathfrak{S}_{n}(\sigma)$ be the set of $\sigma$-avoiding permutations of arbitrary length.

Remark 1.1. For later use, note that $n_{\sigma^{-1}}\left(\pi^{-1}\right)=n_{\sigma}(\pi)$. Similarly, for the reverse $\sigma^{\dagger}:=\sigma_{k} \ldots \sigma_{1}, n_{\sigma^{\dagger}}\left(\pi^{\dagger}\right)=n_{\sigma}(\pi)$, and for the complement $\bar{\sigma}=$ $\left(k+1-\sigma_{1}\right) \cdots\left(k+1-\sigma_{k}\right), n_{\bar{\sigma}}(\bar{\pi})=n_{\sigma}(\pi)$. In particular, the maps $\pi \mapsto \pi^{-1}$, $\pi \mapsto \pi^{\dagger}$ and $\pi \mapsto \bar{\pi}$ are bijections $\mathfrak{S}_{n}(\sigma) \rightarrow \mathfrak{S}_{n}\left(\sigma^{-1}\right), \mathfrak{S}_{n}(\sigma) \rightarrow \mathfrak{S}_{n}\left(\sigma^{\dagger}\right)$ and $\mathfrak{S}_{n}(\sigma) \rightarrow \mathfrak{S}_{n}(\bar{\sigma})$.

The general problem that we are interested in here is to take a fixed permutation $\tau$, and let $\boldsymbol{\pi}_{\tau, n}$ be a uniformly random $\tau$-avoiding permutation, i.e., a uniformly random element of $\mathfrak{S}_{n}(\tau)$, and then study the distribution

Date: 22 January, 2014.

2010 Mathematics Subject Classification. 60C05; 05A05, 60F05.

Partly supported by the Knut and Alice Wallenberg Foundation. 
of the random variable $n_{\sigma}\left(\boldsymbol{\pi}_{\tau, n}\right)$ for some other fixed permutation $\sigma$. More precisely, we are mainly interested in asymptotics of the distribution as $n \rightarrow \infty$. (Although our methods also yield exact formulas for finite $n$.) The present paper is only a partial contribution to this general problem, and we will soon concentrate on the single case $\tau=132$.

Remark 1.2. It is well-known that if $\pi$ is a uniformly random permutation in $\mathfrak{S}_{n}$, without any restriction, and $\sigma$ is a fixed permutation, then $n_{\sigma}(\pi)$ has an asymptotic normal distribution as $n \rightarrow \infty$; moreover, this holds jointly for several $\sigma$. See Bóna [9, 11] and Janson, Nakamura and Zeilberger [25]. We shall see that the restricted case is different.

Remark 1.3. The case $|\tau|=2$ is trivial. By symmetry (Remark 1.1), it suffices to consider $\tau=21$, and then $n_{\tau}(\pi)$ is the number of inversions in $\pi$; the only permutation in $\mathfrak{S}_{n}$ that avoids 21 is the identity permutation so $\mathfrak{S}_{n}(21)=\{12 \cdots n\}$ has only one element. Hence, the simplest non-trivial cases are the cases $|\tau|=3$. There are 6 permutations $\tau \in \mathfrak{S}_{3}$, but by the symmetries in Remark 1.1, it suffices to consider the two cases $\tau=123$ and 132.

As a background, note first that it is a classical problem to enumerate the sets $\mathfrak{S}_{n}(\tau)$, either exactly or asymptotically, and to study various properties of the generating function; see Bóna [8, Chapters 4-5]. In particular, two permutations $\sigma$ and $\tau$ are said to be Wilf-equivalent if $\left|\mathfrak{S}_{n}(\sigma)\right|=\left|\mathfrak{S}_{n}(\tau)\right|$ for all $n$. It is know that all permutations of length 3 are Wilf-equivalent, with $\left|\mathfrak{S}_{n}(\tau)\right|=\left(\begin{array}{c}2 n \\ n\end{array}\right) /(n+1)$, the $n$th Catalan number $C_{n}$, when $|\tau|=3$, see e.g. [27, Exercises 2.2.1-4], [40], [41, Exercise 6.19ee,ff], 8, Corollary 4.7]; in contrast, not all permutations of length 4 are Wilf-equivalent. (The classification of Wilf-equivalent permutations of length 4 was quite difficult, see [8] and the references given there.)

A simpler version of the general problem above is to find (at least asymptotically) the expectation $\mathbb{E} n_{\sigma}\left(\boldsymbol{\pi}_{\tau, n}\right)$. (If the number $\left|\mathfrak{S}_{n}(\tau)\right|$ is known, this is equivalent to finding the total number of occurrences of $\sigma$ in all $\tau$-avoiding permutations of length $n$.) This version of the problem was posed by Cooper [16], and has been studied by Bóna [10] $(\tau=132, \sigma=1 \cdots k$ and $\sigma=k \cdots 1)$, Bóna [12] $(\tau=132,|\sigma|=3$ and certain longer $\sigma)$, Homberger [22] $(\tau=123$, $|\sigma| \leqslant 3$ ); furthermore Cheng, $\mathrm{Eu}$ and $\mathrm{Fu}[14]$ studies the case $\tau=321$, $\sigma=21$. (or, equivalently, $\tau=123, \sigma=12$ ). These papers concentrate on exact formulas and generating functions; asymptotics are derived as corollaries. Rudolph [39] studied the problem of when $\mathbb{E} n_{\sigma_{1}}\left(\boldsymbol{\pi}_{\tau, n}\right)=\mathbb{E} n_{\sigma_{2}}\left(\boldsymbol{\pi}_{\tau, n}\right)$ (in the case $\tau=132$ ).

In particular, for $\tau=132$, by [10], [12] and straightforward singularity analysis (see [20, Chapter VI]), or by Examples $[5.8$ and $[5.12$ below, as $n \rightarrow \infty$,

$$
\mathbb{E} n_{12}\left(\boldsymbol{\pi}_{132, n}\right) \sim \frac{\sqrt{\pi}}{2} n^{3 / 2},
$$




$$
\begin{aligned}
& \mathbb{E} n_{123}\left(\boldsymbol{\pi}_{132, n}\right) \sim \frac{1}{2} n^{2}, \\
& \mathbb{E} n_{213}\left(\boldsymbol{\pi}_{132, n}\right)=\mathbb{E} n_{231}\left(\boldsymbol{\pi}_{132, n}\right)=\mathbb{E} n_{312}\left(\boldsymbol{\pi}_{132, n}\right) \sim \frac{\sqrt{\pi}}{8} n^{5 / 2}, \\
& \mathbb{E} n_{321}\left(\boldsymbol{\pi}_{132, n}\right) \sim\left(\begin{array}{l}
n \\
3
\end{array}\right) \sim \frac{1}{6} n^{3},
\end{aligned}
$$

and, for any fixed $k \geqslant 1$, generalizing (1.3)-(1.4),

$$
\mathbb{E} n_{1 \ldots k}\left(\boldsymbol{\pi}_{132, n}\right) \sim \frac{2^{1-k} \sqrt{\pi}}{\Gamma(k / 2)} n^{(k+1) / 2} .
$$

Note that in (1.5), the three expectations are equal for any $n$; the equality of the two latter is trivial because $n_{231}\left(\boldsymbol{\pi}_{132, n}\right)$ and $n_{312}\left(\boldsymbol{\pi}_{132, n}\right)$ have the same distribution, as a consequence of the first symmetry in Remark 1.1. The first equality is non-trivial and more surprising; in fact $n_{213}\left(\boldsymbol{\pi}_{132, n}\right)$ and $n_{231}\left(\boldsymbol{\pi}_{132, n}\right)$ do not have the same distribution, in general. (They have different variances already for $n=5$, as is shown by an enumeration, by hand or by computer.)

The more general problem of studying the distribution, and not just the expectation, of $n_{\sigma}\left(\boldsymbol{\pi}_{\tau, n}\right)$ was raised in [25], where higher moments (and mixed moments) are calculated (using computer algebra) for small $n$ for several cases $(\tau=132,123$ and 1234; several $\sigma$ with $|\sigma|=3)$.

The main result of the present paper (Section 2) is that the formulas above for the expectation generalize to arbitrary $\sigma \in \mathfrak{S}_{*}(132)$, always with growth as a half-integer power of $n$, and that, moreover, the random variables after normalization by this power of $n$ converge to some positive limit random variables, with convergence of all moments.

Remark 1.4. The case of forbidding $\tau=123$ has, as said above, been studied by Cheng, Eu and Fu [14] $(\sigma=12)$ and Homberger [22] $(|\sigma| \leqslant 3)$; their results yield (after simple calculations and corrections of several typos in [22]), as $n \rightarrow \infty$,

$$
\begin{aligned}
\mathbb{E} n_{12}\left(\boldsymbol{\pi}_{123, n}\right) & \sim \frac{\sqrt{\pi}}{4} n^{3 / 2}, \\
\mathbb{E} n_{132}\left(\boldsymbol{\pi}_{123, n}\right) & =\mathbb{E} n_{213}\left(\boldsymbol{\pi}_{123, n}\right) \sim \frac{1}{4} n^{2}, \\
\mathbb{E} n_{231}\left(\boldsymbol{\pi}_{123, n}\right) & =\mathbb{E} n_{312}\left(\boldsymbol{\pi}_{123, n}\right) \sim \frac{\sqrt{\pi}}{8} n^{5 / 2},
\end{aligned}
$$

and, which also follows from these and (1.1),

$$
\mathbb{E} n_{321}\left(\boldsymbol{\pi}_{123, n}\right) \sim\left(\begin{array}{l}
n \\
3
\end{array}\right) \sim \frac{1}{6} n^{3} .
$$

Cf. (1.3)-(1.6). Moreover, Homberger [22] shows that also $\mathbb{E} n_{231}\left(\boldsymbol{\pi}_{123, n}\right)=$ $\mathbb{E} n_{231}\left(\boldsymbol{\pi}_{132, n}\right)$ for any $n$; however, the distribution of $n_{231}\left(\boldsymbol{\pi}_{123, n}\right)$ differs (in 
general) from the distribution of any of the variables in (1.5). (They have different variances already for $n=4$.)

The equivalence given by [14] between $n_{12}\left(\boldsymbol{\pi}_{123, n}\right)$ and the number of certain squares under a Catalan path (or equivalently, a Dyck path) implies by standard results that

$$
n^{-1 / 2} n_{12}\left(\boldsymbol{\pi}_{123, n}\right) \sim 2^{-1 / 2} \int_{0}^{1} \mathbf{e}(x) \mathrm{d} x
$$

where $\mathbf{e}$ is a Brownian excursion; this is apart from a factor $1 / 2$ the same limit as for $n_{12}\left(\boldsymbol{\pi}_{132, n}\right)$, see Example 7.6. For the other cases above (excluding the trivial $n_{321}$ ) we do not know any asymptotic distribution, and not even asymptotic second moments. It seems likely that methods similar to the present paper could be useful in this case too, using a suitable bijection between $\mathfrak{S}_{n}(123)$ and binary trees (cf. Remark 4.3), but we have not yet attempted it.

It seems much more difficult to show results for any longer $\tau$.

Remark 1.5. A special case of the distribution of $n_{\sigma}\left(\boldsymbol{\pi}_{\tau, n}\right)$ is the probability $\mathbb{P}\left(n_{\sigma}\left(\boldsymbol{\pi}_{\tau, n}\right)=0\right)$ that a $\tau$-avoiding permutation also avoids $\sigma$; this is equivalent to enumerating the set $\mathfrak{S}_{n}(\sigma, \tau)$ of permutations that avoid both $\sigma$ and $\tau$ (given that we know $\left|\mathfrak{S}_{n}(\tau)\right|$ ). This problem has been studied by various authors (with exact results, generating functions and asymptotics), see e.g. [40], [6], [42], [15], 38], 30], [31], [32], 28], [1], [2]. Some of these also consider the number of $\tau$-avoiding permutations with exactly $r$ occurences of $\sigma$, which is equivalent to $\mathbb{P}\left(n_{\sigma}\left(\boldsymbol{\pi}_{\tau, n}\right)=r\right)$. Formally, this is the same as our problem of the distribution of $n_{\sigma}\left(\boldsymbol{\pi}_{\tau, n}\right)$, but the emphasis in these papers is on exact formulas for constant $r$, while we are interested in asymptotic results, with $r$ increasing. It would be interesting to derive asymptotic distributions from these algebraic results, but this seems difficult.

Remark 1.6. We have considered avoiding a single pattern $\tau$. Of course, the same questions can be asked for a set $\tau_{1}, \ldots, \tau_{M}$ of two or several forbidden patterns, cf. the references in Remark 1.5 where such sets $\mathfrak{S}_{n}\left(\tau_{1}, \ldots, \tau_{M}\right)$ are studied. For a simple example, there are exactly $2^{n-1}$ permutations in $\mathfrak{S}_{n}(123,132)$, and they have a simple structure [40] which makes it easy to see that the number $n_{12}$ of noninversions has a binomial distribution $\operatorname{Bi}(n-$ $1,1 / 2)$; in this case, $n_{12}$ thus has an asymptotically normal distribution.

\section{MAin Results}

From now on we consider only $\tau=132$. Note that $n_{\sigma}\left(\boldsymbol{\pi}_{132, n}\right)=0$ if $\sigma$ contains a copy of $\tau$; hence we only consider $\sigma$ that themselves avoid $\tau$.

Recall that a descent in a permutation $\sigma_{1} \cdots \sigma_{k}$ is an index $i \in[k-1]$ such that $\sigma_{i}>\sigma_{i+1}$; we also define the last index $k$ to be a decent. (Tradition varies about the latter case; we find this version convenient for our purposes.) We let $D(\sigma)$ be the number of descents in $\sigma$. (Note that with our definition 
$1 \leqslant D(\sigma) \leqslant|\sigma|$.$) We define$

$$
\lambda(\sigma):=|\sigma|+D(\sigma)
$$

and note that

$$
|\sigma|+1 \leqslant \lambda(\sigma) \leqslant 2|\sigma|
$$

with the extreme values $\lambda(\sigma)=|\sigma|+1$ if and only if $\sigma=1 \cdots k$, and $\lambda(\sigma)=2|\sigma|$ if and only if $\sigma=k \cdots 1$, where $k=|\sigma|$.

Theorem 2.1. There exist strictly positive random variables $\Lambda_{\sigma}$ such that

$$
n_{\sigma}\left(\boldsymbol{\pi}_{132, n}\right) / n^{\lambda(\sigma) / 2} \stackrel{\mathrm{d}}{\longrightarrow} \Lambda_{\sigma},
$$

as $n \rightarrow \infty$, jointly for all $\sigma \in \mathfrak{S}_{*}(132)$. Moreover, this holds with convergence of all moments, with all moments of $\Lambda_{\sigma}$ finite, i.e., for any sequence $\sigma^{(1)}, \ldots, \sigma^{(M)} \in \mathfrak{S}_{*}(132)$, possibly with repetitions,

$$
\mathbb{E}\left(n_{\sigma^{(1)}} \cdots n_{\sigma^{(M)}}\left(\boldsymbol{\pi}_{132, n}\right)\right) \sim n^{\sum_{\nu} \lambda\left(\sigma^{(\nu)}\right) / 2} \mathbb{E}\left(\Lambda_{\sigma^{(1)}} \cdots \Lambda_{\sigma^{(M)}}\right) .
$$

In particular, for every $\sigma \in \mathfrak{S}_{*}(132)$, there exists a positive constant $A_{\sigma}=$ $\mathbb{E} \Lambda_{\sigma}$ such that

$$
\mathbb{E} n_{\sigma}\left(\boldsymbol{\pi}_{132, n}\right) \sim A_{\sigma} n^{\lambda(\sigma) / 2} .
$$

For a monotone decreasing permutation $k \cdots 1, \Lambda_{k \cdots 1}=1 / k$ ! is deterministic, but not for any other $\sigma$.

Remark 2.2. Since $\Lambda_{\sigma}>0$, the limit distributions are not normal; thus $n_{\sigma}\left(\boldsymbol{\pi}_{132, n}\right)$ is not asymptotically normal. (For $\sigma=k \cdots 1$, use (2.12) below.) This was conjectured (for $\sigma=312$ ) in [25] based on calculation of the moments for small $n$; our theorem verifies this, but it should be noted that the numerical values in [25, Table 3] for $n \leqslant 20$ are still far from their limits. A calculation using Theorem 6.5 shows that the normalized third moment $\mathbb{E}(X-\mathbb{E} X)^{3} / \operatorname{Var}(X)^{3 / 2} \approx 0.76384$ for the limit $X=\Lambda_{312}$, while for $n=20$, [25] yields 0.44906 .

The proof of Theorem 2.1 will occupy the rest of the paper. We will use two completely different methods that complement each other and prove different parts of the theorem; both use a bijection with binary trees described in Section 4. One method (Section 7 and Theorem 7.5) uses this to show the convergence in distribution (2.3); this proof shows also that the limit random variables $\Lambda_{\sigma}$ can be expressed as functionals of a Brownian excursion $\mathbf{e}(x)$. In particular (Example 7.6),$\Lambda_{12}=\sqrt{2} \int_{0}^{1} \mathbf{e}(x) \mathrm{d} x$; this is (apart from the factor $\sqrt{2}$ ) the well-known Brownian excursion area which appears as a limit in various combinatorial problems (for instance for the total path length in a random conditioned Galton-Watson tree [3; 4]); for this distribution see also the survey [24] and the references there. (It is sometimes called the Airy distribution.) More generally (Example 7.8), for the monotone pattern $1 \cdots k, \Lambda_{1 \cdots k}=c_{k} \int_{0}^{1} \mathbf{e}(x)^{k-1} \mathrm{~d} x$ with $c_{k}=2^{(k-1) / 2} /(k-1)$ !. However, in general, the description as a Brownian excursion functional is rather complicated, and it is not easy to even compute its mean. 
As a complement, we therefore give also by another method (Section 5) formulas yielding (by recursion) the constants $A_{\sigma}=\mathbb{E} \Lambda_{\sigma}$, see (5.19) and (5.8); we describe also (Section 6) how one can similarly find also limits for higher moments (possibly mixed). This method uses a recursion for the numbers $n_{\sigma}(\pi)$ that is given in Section 3, and a probabilistic argument using subcritical Galton-Watson trees. As examples, we give (Theorem 6.5) explicit recursion relations for the moments of $\Lambda_{\sigma}$ for $|\sigma| \leqslant 3$ (and joint moments of $\Lambda_{12}$ and $\Lambda_{\sigma}$ with $|\sigma|=3$, needed for the recursions). In particular, Theorem 6.5 yields for the second moments (where (2.6) is well-known, see [29], 24])

$$
\begin{array}{rlrl}
\mathbb{E} \Lambda_{12}^{2} & =\frac{5}{6}, & \operatorname{Var} \Lambda_{12} & =\frac{10-3 \pi}{12}, \\
\mathbb{E} \Lambda_{123}^{2} & =\frac{19}{60}, & \operatorname{Var} \Lambda_{123} & =\frac{1}{15}, \\
\mathbb{E} \Lambda_{213}^{2} & =\frac{7}{120}, & \operatorname{Var} \Lambda_{213} & =\frac{56-15 \pi}{960}, \\
\mathbb{E} \Lambda_{231}^{2}= & \mathbb{E} \Lambda_{312}^{2}=\frac{43}{840}, & \operatorname{Var} \Lambda_{231}=\operatorname{Var} \Lambda_{312}=\frac{344-105 \pi}{6720} .
\end{array}
$$

For mixed moments we find from Theorem 6.5 for example

$$
\begin{array}{r}
\mathbb{E}\left(\Lambda_{12} \Lambda_{213}\right)=\frac{13}{60}, \\
\mathbb{E}\left(\Lambda_{12} \Lambda_{231}\right)=\mathbb{E}\left(\Lambda_{12} \Lambda_{312}\right)=\frac{1}{5} .
\end{array}
$$

The matrix of second moments of $\left(\Lambda_{213}, \Lambda_{231}, \Lambda_{312}\right)$ is given in (6.19).

Remark 2.3. For a given $|\sigma|=k$, we see that the order of $\mathbb{E} n_{\sigma}\left(\boldsymbol{\pi}_{132, n}\right)$ is smallest $\left(n^{(k+1) / 2}\right)$ for $\sigma=1 \cdots k$ and largest $\left(n^{k}\right)$ for $\sigma=k \cdots 1$. Cf. the related result by Bóna [10] that for every $n, \mathbb{E} n_{1 \cdots k}\left(\boldsymbol{\pi}_{132, n}\right) \leqslant \mathbb{E} n_{\sigma}\left(\boldsymbol{\pi}_{132, n}\right) \leqslant$ $\mathbb{E} n_{k \cdots 1}\left(\boldsymbol{\pi}_{132, n}\right)$ for every $\sigma \in \mathfrak{S}_{k}(132)$, see Section 9 .

Remark 2.4. In particular, (2.5) implies that $\mathbb{E} n_{\sigma}\left(\boldsymbol{\pi}_{132, n}\right) / n^{k} \rightarrow 0$ for every $\sigma \in \mathfrak{S}_{k}$ except $k \cdots 1$, which by (1.1) trivially implies $\mathbb{E} n_{k \cdots 1}\left(\boldsymbol{\pi}_{132, n}\right) \sim\left(\begin{array}{l}n \\ k\end{array}\right)$ and $n_{k \cdots 1}\left(\boldsymbol{\pi}_{132, n}\right) \stackrel{\mathrm{p}}{\longrightarrow} 1 / k$ !, which is the case $\sigma=k \cdots 1$ of Theorem 2.1 with $\Lambda_{k \cdots 1}=1 / k$ ! deterministic as asserted in the theorem.

For a nondegenerate limit law also in this case (for $k>1$ ), note that the same argument yields

$$
n^{-(k-1 / 2)}\left(\left(\begin{array}{l}
n \\
k
\end{array}\right)-n_{k \cdots 1}\left(\boldsymbol{\pi}_{132, n}\right)\right) \stackrel{\mathrm{d}}{\longrightarrow} \sum \Lambda_{\sigma},
$$

summing over all $\sigma \in \mathfrak{S}_{k}(132)$ with $\lambda(\sigma)=2 k-1$ (i.e., $D(\sigma)=k-1$ ).

Remark 2.5. Although the exponent in (2.5) depends only on $\lambda(\sigma)$, i.e., on $|\sigma|$ and the number of descents in $\sigma$, the constant $A_{\sigma}$ does not. For example, it follows by (5.19) and (5.8), or by Example 5.11 and Lemma 5.4, that $\mathbb{E} n_{3214} \sim \frac{\sqrt{\pi}}{32} n^{7 / 2}$ and $\mathbb{E} n_{3241} \sim \frac{\sqrt{\pi}}{64} n^{7 / 2}$. 
Remark 2.6. Apart from the relation (1.1), there are also simple relations between the counts $n_{\sigma}(\pi)$ for $\sigma$ of different lengths. For example,

$$
(n-2) n_{12}(\pi)=3 n_{123}(\pi)+2 n_{132}(\pi)+2 n_{213}(\pi)+n_{231}(\pi)+n_{312}(\pi),
$$

since the left-hand side counts the number of distinct $i, j, k$ such that $i<j$ and $\pi_{i}<\pi_{j}$, and if $\sigma \in \mathfrak{S}_{3}$, then each occurence of $\sigma$ in $\pi$ contributes $n_{12}(\sigma)$ such triples.

For $\pi \in \mathfrak{S}_{n}(132)$, the term $n_{132}(\pi)$ vanishes, and if we divide by $n^{5 / 2}$ and take the limit, another term disappears asymptotically, and we find for the limit variables the relation

$$
\Lambda_{12}=2 \Lambda_{213}+\Lambda_{231}+\Lambda_{312}
$$

Similar relations enable each $\Lambda_{\sigma}$ to be expressed in $\Lambda_{\sigma^{\prime}}$ for some set of $\sigma^{\prime}$ with $\left|\sigma^{\prime}\right|=|\sigma|+1$.

Remark 2.7. The limit $\Lambda_{213}$ and the sum $\Lambda_{231}+\Lambda_{312}$ have appeared earlier as distribution limits in [23], see Remark 7.12.

\section{A BASIC RECURSION}

If $x_{1} \cdots x_{n}$ is any sequence of distinct numbers, let $\Pi\left(x_{1} \cdots x_{n}\right)$ be the permutation in $\mathfrak{S}_{n}$ that has the same order as $x_{1} \cdots x_{n}$. We extend the notation $n_{\sigma}(\pi)$ in the trivial way to arbitrary sequences of distinct numbers $x_{1} \cdots x_{n}$ and $y_{1} \cdots y_{k}$ by $n_{y_{1} \cdots y_{k}}\left(x_{1} \cdots x_{n}\right):=n_{\Pi\left(y_{1} \cdots y_{k}\right)}\left(\Pi\left(x_{1} \cdots x_{n}\right)\right)$. (We may similarly extend other notations when convenient.) We also define $n_{\emptyset}\left(x_{1} \cdots x_{n}\right)=0$ for an empty string $\emptyset$ (i.e., the case $k=0$ ), and let $\mathfrak{S}_{0}:=$ $\{\emptyset\}$.

If $\pi \in \mathfrak{S}_{n}$ and $\ell$ is the index of the maximal element $n$, i.e.. $\pi_{\ell}=n$, let $\pi_{L}:=\pi_{1} \cdots \pi_{\ell-1}$ and $\pi_{R}:=\pi_{\ell+1} \cdots \pi_{n}$ be the (possibly empty) parts of $\pi$ before and after the maximal element. Using the operator $\Pi$ above, we can regard them as permutations $\pi_{L} \in \mathfrak{S}_{\ell-1}$ and $\pi_{R} \in \mathfrak{S}_{n-\ell}$.

We begin with a well-known characterization of the 132-avoiding permutations, see e.g. Bóna [10].

Lemma 3.1. With notations as above, a permutation $\pi$ avoids 132 if and only if $\pi_{L}$ and $\pi_{R}$ both avoid 132 and furthermore $\pi_{i}>\pi_{j}$ whenever $i<\ell$ and $j>\ell$.

Proof. Although this is well-known and easy, we sketch the proof for completeness.

If $\pi$ avoids 132 then so do $\pi_{L}$ and $\pi_{R}$. Furthermore, if the final condition in the lemma is violated, then $\pi_{i}<\pi_{j}<\pi_{\ell}$ for some $i$ and $j$ with $i<\ell<j$, and thus $\pi_{i} \pi_{\ell} \pi_{j}$ is an occurrence of 132 .

The converse is just as easy, by considering the possible positions of an occurrence of 132 in relation to $\ell$; we omit the details.

This leads to a basic recursion for $n_{\sigma}(\pi)$. 
Lemma 3.2. Let $\sigma \in \mathfrak{S}_{k}(132)$ with $k \geqslant 1$. Define $m$ by $\sigma_{m}=k$ and let $\Delta:=\left\{q \in[k-1]: \min _{1 \leqslant i \leqslant q} \sigma_{i}>\max _{q<j \leqslant k} \sigma_{j}\right\}$. Then, for any permutation $\pi \in \mathfrak{S}_{n}(132)$ with $n \geqslant 1$,

$$
\begin{aligned}
n_{\sigma}(\pi)=n_{\sigma}\left(\pi_{L}\right)+n_{\sigma}\left(\pi_{R}\right)+\sum_{q \in \Delta} n_{\sigma_{1} \cdots \sigma_{q}}\left(\pi_{L}\right) n_{\sigma_{q+1} \cdots \sigma_{k}}\left(\pi_{R}\right) & \\
& +n_{\sigma_{1} \cdots \sigma_{m-1}}\left(\pi_{L}\right) n_{\sigma_{m+1} \cdots \sigma_{k}}\left(\pi_{R}\right) .
\end{aligned}
$$

Proof. Consider first an occurrence $\pi_{\nu_{1}} \cdots \pi_{\nu_{k}}$ of $\sigma$ that does not include $\pi_{\ell}$. Then, for some $q \in\{0, \ldots, k\}, \nu_{1}<\cdots<\nu_{q}<\ell<\nu_{q+1}<\cdots<\nu_{k}$.

The cases $q=k$ and $q=0$ give the $n_{\sigma}\left(\pi_{L}\right)$ and $n_{\sigma}\left(\pi_{R}\right)$ occurrences in $\pi_{L}$ and $\pi_{R}$.

If $1 \leqslant q \leqslant k-1$, we note that by Lemma 3.1, if $i \leqslant q$ and $j>q$, then $\pi_{\nu_{i}}>\pi_{\nu_{j}}$ and thus $\sigma_{i}>\sigma_{j}$; hence $q \in \Delta$. Furthermore, for every $q \in \Delta$, we have excatly one such occurrence $\sigma$ in $\pi$ for every pair of occurrences of $\sigma_{1} \cdots \sigma_{q}$ in $\pi_{L}$ and $\sigma_{q+1} \cdots \sigma_{k}$ in $\pi_{R}$. The total number of such occurrences is thus the sum in (3.1).

Finally, if an occurrence $\pi_{\nu_{1}} \cdots \pi_{\nu_{k}}$ of $\sigma$ contains $\pi_{\ell}=n$, then $\pi_{\ell}$ must correspond to the largest element $\sigma_{m}$ in $\sigma$, i.e. $\nu_{m}=\ell$. It follows in the same way as above that the number of such occurrences is $n_{\sigma_{1} \cdots \sigma_{m-1}}\left(\pi_{L}\right) n_{\sigma_{m+1} \cdots \sigma_{k}}\left(\pi_{R}\right)$.

The set $\Delta$ is empty if $m=k$; otherwise $m \in \Delta$ by Lemma 3.1 so $\Delta \neq \emptyset$. The extreme case is $\sigma=k \cdots 1$ when $\Delta=[k-1]$. Note that every element of $\Delta$ is a descent in $\sigma$ (but not conversely, in general).

\section{Binary trees}

Out proofs are based on a well-known bijection between $\mathfrak{S}_{n}(132)$ and the set $\mathcal{B}_{n}$ of binary trees of order $n$, see e.g. [12]. It can be defined as follows.

Recall that a binary tree $T$ consist of a root and two subtrees $T_{L}$ and $T_{R}$ (the left and right subtree) which are either empty or themselves binary trees. Using the notations of Section 3, we define recursively for any permutation $\pi \in \mathfrak{S}_{n}(132)$ with $n \geqslant 1$ a binary tree $T=T(\pi) \in \mathcal{B}_{n}$ such that its left subtree $T_{L}=T\left(\pi_{L}\right)$ and its right subtree $T_{R}=T\left(\pi_{R}\right)$; furthermore, $T(\emptyset)$ is the empty tree. It is easy to see that this yields a bijection between $\mathfrak{S}_{n}(132)$ and $\mathcal{B}_{n}$.

If $T$ is a binary tree, and $\sigma$ is a permutation, let $X_{\sigma}(T):=n_{\sigma}\left(\pi_{T}\right)$, where $\pi_{T} \in \mathfrak{S}(132)$ is the permutation corresponding to $T$ by the bijection above. Moreover, let $X_{\sigma, L}:=X_{\sigma}\left(T_{L}\right)$ and $X_{\sigma, R}:=X_{\sigma}\left(T_{R}\right)$, where $L$ and $R$ are the left and right subtrees of $T$.

We can translate the recursion Lemma 3.2 to recursive relations for the variables $X_{\sigma}=X_{\sigma}(T)$ as follows. (We usually omit the $\operatorname{argument} T$ for notational convenience.)

Lemma 4.1. Let $\sigma \in \mathfrak{S}_{k}(132)$ with $k \geqslant 1$ and define $m$ and $\Delta$ as in Lemma 3.2. Then, for any binary tree $T$, 


$$
\begin{aligned}
X_{\sigma}=X_{\sigma, L}+X_{\sigma, R}+\sum_{q \in \Delta} X_{\sigma_{1} \cdots \sigma_{q}, L} X_{\sigma_{q+1} \cdots \sigma_{k}, R} & \\
& +X_{\sigma_{1} \cdots \sigma_{m-1}, L} X_{\sigma_{m+1} \cdots \sigma_{k}, R}
\end{aligned}
$$

Note also that $X_{\sigma}=0$ unless $\sigma \in \mathfrak{S}_{*}(132)$ and, by (1.1),

$$
\sum_{\sigma \in \mathfrak{S}_{k}} X_{\sigma}=\left(\begin{array}{l}
n \\
k
\end{array}\right) .
$$

As an illustration and for later use, we write the recursion (4.1) explicitly for some small $\sigma$. For (notational) convenience, we define $N=N(T):=$ $X_{1}(T)=|T|$ and $Y=Y(T):=X_{12}(T)$, and define $N_{L}, N_{R}, Y_{L}, Y_{R}$ correspondingly. Note that then, by (4.2),

$$
X_{21}=\left(\begin{array}{c}
N \\
2
\end{array}\right)-X_{12}=\left(\begin{array}{c}
N \\
2
\end{array}\right)-Y .
$$

Example 4.2. Taking $\sigma=1,12,123,213,231,312$ in Lemma 4.1 we find the following recursions, noting that in these cases $\Delta=\Delta_{\sigma}$ is empty except $\Delta_{231}=\{2\}$ and $\Delta_{312}=\{1\}$; for (4.7) we use also (4.3).

$$
\begin{aligned}
N & =N_{L}+N_{R}+1, \\
Y & =Y_{L}+Y_{R}+N_{L}, \\
X_{123} & =X_{123, L}+X_{123, R}+Y_{L}, \\
X_{213} & =X_{213, L}+X_{213, R}+\left(\begin{array}{c}
N_{L} \\
2
\end{array}\right)-Y_{L}, \\
X_{231} & =X_{231, L}+X_{231, R}+Y_{L} N_{R}+N_{L} N_{R}, \\
X_{312} & =X_{312, L}+X_{312, R}+N_{L} Y_{R}+Y_{R} .
\end{aligned}
$$

(These recursions can also easily be verified directly, and (4.4) is utterly trivial.)

Let $T_{n}$ be a uniformly random binary tree in $\mathcal{B}_{n}$. Note that $T_{n}$ by the bijection above corresponds to a uniformly random permutation in $\mathfrak{S}_{n}(132)$, i.e. we can identify $T_{n}=T\left(\boldsymbol{\pi}_{132, n}\right)$. With this identification and the notations above we have

$$
X_{\sigma}\left(T_{n}\right)=n_{\sigma}\left(\boldsymbol{\pi}_{132, n}\right)
$$

we will in the sequel use this without comment and study the random variables $X_{\sigma}\left(T_{n}\right)$ when proving Theorem 2.1.

Remark 4.3. The bijection with $\mathcal{B}_{n}$ is equivalent to a bijection with the set of Dyck paths of length $2 n$, by the well-known standard bijection between the latter and $\mathcal{B}_{n}$. This is equivalent to the bijection by Knuth [27, Exercises 2.2.1-3,5] between 312-avoiding permutations and Dyck paths. Another bijection with Dyck paths is given by 28]. For similar bijections of $\mathfrak{S}_{n}(123)$ and Dyck paths, see e.g. [6], [28], [14]. See also the many bijections with various objects in Stanley [41, Exercise 6.19 (and its solution)]. 


\section{Expectations}

We next use an idea from [23] and consider the functionals $X_{\sigma}$ above for another random binary tree $T_{\delta}$ defined as follows, for $0<\delta<1$. Note that this random tree, unlike $T_{n}$, has a random size.

We start with the root; we then add each of the two possible children of the root with probability $p:=(1-\delta) / 2$ each, and we continue in the same way with the possible children of any node that we add to the tree, with all random choices independent. Thus $T_{\delta}$ is a random Galton-Watson tree with offspring distribution $\operatorname{Bi}(2, p)$. Since this offspring distribution has expectation $2 p=1-\delta<1$, the Galton-Watson tree $T_{\delta}$ is subcritical and thus a.s. finite.

The construction implies that if $T=T_{\delta}$, then the subtrees $T_{L}$ and $T_{R}$ are independent random trees; furthermore, each of them empty with probability $1-p=(1+\delta) / 2$ and otherwise it has the same distribution as $T$. (This can be used as an alternative, recursive definition of $T_{\delta}$.)

Remark 5.1. The argument in [23] uses full binary trees, which makes the details a little different although the main idea is the same. We thus present the argument in detail below, and refer the interested reader to [23] for comparisons.

We let $\mathbb{E}_{\delta}$ denote expectation of random variables defined for the random tree $T=T_{\delta}$. These expectations are generating functions in disguise. In fact, let $Z=Z(T)$ be an arbitrary functional such that $|Z(T)| \leqslant C|T|^{m}$ for some constants $C$ and $m$. (This guarantees that all expectations and sums below converge, and is satisfied by the functionals that we consider, viz. $X_{\sigma}$ and products of these.) We write $z_{n}:=\mathbb{E} Z\left(T_{n}\right)$.

Lemma 5.2. Let $Z$ and $z_{n}:=\mathbb{E} Z\left(T_{n}\right)$ be as above. Then

$$
\mathbb{E}_{\delta} Z=\frac{1+\delta}{1-\delta} \sum_{n=1}^{\infty} z_{n} C_{n}\left(\frac{1-\delta^{2}}{4}\right)^{n} .
$$

Proof. There are $C_{n}=\left(\begin{array}{c}2 n \\ n\end{array}\right) /(n+1)$ trees in $\mathcal{B}_{n}$. If $T \in \mathcal{B}_{n}$, then $T$ has $n$ nodes, with 2 potential children each. Of these $2 n$ potential children, $n-1$ exist and $n+1$ do not exist. The probability that $T_{\delta}$ equals a given tree $T \in \mathcal{B}_{n}$ is thus

$$
\mathbb{P}\left(T_{\delta}=T\right)=p^{n-1}(1-p)^{n+1}=2^{-2 n}(1-\delta)^{n-1}(1+\delta)^{n+1} .
$$

This probability is the same for all $T \in \mathcal{B}_{n}$, and since $\left|\mathcal{B}_{n}\right|=C_{n}$, it follows that the probability that $T_{\delta}$ has order $n$ is

$\mathbb{P}\left(\left|T_{\delta}\right|=n\right)=\mathbb{P}\left(T_{\delta} \in \mathcal{B}_{n}\right)=C_{n} 2^{-2 n}(1-\delta)^{n-1}(1+\delta)^{n+1}=C_{n} \frac{1+\delta}{1-\delta}\left(\frac{1-\delta^{2}}{4}\right)^{n}$.

Moreover, since (5.2) does not depend on the choice of $T \in \mathcal{B}_{n}$, we see that conditioned on $\left|T_{\delta}\right|=n, T_{\delta}$ is uniformly distributed in $\mathcal{B}_{n}$; in other words 
$\left(T_{\delta}|| T_{\delta} \mid=n\right) \stackrel{\mathrm{d}}{=} T_{n}$. Hence, $\mathbb{E}\left(Z|| T_{\delta} \mid=n\right)=\mathbb{E} Z\left(T_{n}\right)=z_{n}$ and, using (5.3),

$$
\begin{aligned}
\mathbb{E}_{\delta} Z & =\sum_{n=1}^{\infty} \mathbb{P}\left(\left|T_{\delta}\right|=n\right) \mathbb{E}\left(Z|| T_{\delta} \mid=n\right)=\sum_{n=1}^{\infty} \mathbb{P}\left(\left|T_{\delta}\right|=n\right) z_{n} \\
& =\sum_{n=1}^{\infty} z_{n} C_{n} \frac{1+\delta}{1-\delta}\left(\frac{1-\delta^{2}}{4}\right)^{n} .
\end{aligned}
$$

By Lemma $5.2, \mathbb{E}_{\delta} Z$ is, apart from the factor $(1+\delta) /(1-\delta)$, the ordinary generating function of the sequence $C_{n} z_{n}$, evaluated at $\left(1-\delta^{2}\right) / 4$. Conversely, by taking $\delta=\sqrt{1-4 x}$ in (5.1), we obtain, for $0<x<1 / 4$,

$$
\sum_{n=1}^{\infty} C_{n} z_{n} x^{n}=\frac{1-\sqrt{1-4 x}}{1+\sqrt{1-4 x}} \mathbb{E}_{\sqrt{1-4 x}} Z=\frac{1-2 x-\sqrt{1-4 x}}{2 x} \mathbb{E}_{\sqrt{1-4 x}} Z
$$

Note that $Z=1$ yields the well-known generating function for the Catalan numbers, see e.g. [20, p. 35].

Remark 5.3. For the variables $Z$ that we study below (products of $X_{\sigma}$ ), $\mathbb{E}_{\delta} Z$ turns out to be a polynomial in $\delta^{-1}$; in this case (5.4) yields the generating function $\sum_{n=1}^{\infty} C_{n} z_{n} x^{n}$ as a rational function of $\sqrt{1-4 x}$. By analytic continuation, the resulting formula is valid for all complex $x$ with $|x|<1 / 4$, and the generating function extends to an analytic function in $\mathbb{C} \backslash[1 / 4, \infty)$.

We can now apply singularity analysis and obtain asymptotics of $z_{n}$ from asymptotics of $\mathbb{E}_{\delta} Z$ as $\delta \searrow 0$. (Note that although we can define the random tree $T_{\delta}$ for $\delta=0$, which will be a critical Galton-Watson tree and thus a.s. finite, the expectations that we are interested will all be infinite and of no use to us; hence we consider $\delta>0$ and take asymptotics.) We state a simple case that is enough for our purposes. We let in this section (and the next) $O\left(\delta^{-m}\right)$ denote an arbitrary polynomial in $\delta^{-1}$ of degree at most $m$.

Lemma 5.4. If $\mathbb{E}_{\delta} Z=a \delta^{-m}+O\left(\delta^{-(m-1)}\right)$, where $m \geqslant 1$ and $a \neq 0$, then

$$
\mathbb{E} Z\left(T_{n}\right) \sim a \frac{\Gamma(1 / 2)}{\Gamma(m / 2)} n^{(m+1) / 2} \quad \text { as } n \rightarrow \infty .
$$

Proof. By Remark 5.3, the generating function $\sum_{n=1}^{\infty} C_{n} z_{n} x^{n}$ extends to an analytic function in $\mathbb{C} \backslash[1 / 4, \infty)$, and as $x \rightarrow 1 / 4$, by assumption and (5.4),

$$
\sum_{n=1}^{\infty} c_{n} z_{n} x^{n} \sim a \frac{1-\sqrt{1-4 x}}{1+\sqrt{1-4 x}}(1-4 x)^{-m / 2} \sim a(1-4 x)^{-m / 2} .
$$

This implies by standard singularity analysis (see [20, Corollary VI.1]),

$$
c_{n} z_{n} \sim a 4^{n} \frac{n^{m / 2-1}}{\Gamma(m / 2)} .
$$

The result follows by this and the standard asymptotic expression $C_{n} \sim$ $4^{n} / \sqrt{\pi n^{3}}$ for the Catalan numbers [20, p. 38]. 
For later use, we show also the following, recalling $N(T):=|T|$.

Lemma 5.5. (i) Let $f(\delta)=\mathbb{E}_{\delta} Z$. Then

$$
\mathbb{E}_{\delta}(N Z)=-\frac{1}{2}\left(\delta^{-1}-\delta\right) f^{\prime}(\delta)+\delta^{-1} f(\delta) .
$$

(ii) In particular, if $\mathbb{E}_{\delta} Z=a \delta^{-m}+O\left(\delta^{-(m-1)}\right)$, where $m \geqslant 1$ and $a \in \mathbb{R}$, then $\mathbb{E}_{\delta}(N Z)=\frac{1}{2} m a \delta^{-(m+2)}+O\left(\delta^{-(m+1)}\right)$.

Proof. (i): Differentiate (5.1). This gives, using (5.1) also for $N Z$,

$$
\begin{aligned}
\frac{\mathrm{d}}{\mathrm{d} \delta} \mathbb{E}_{\delta} Z & =\frac{1}{1+\delta} \mathbb{E}_{\delta} Z+\frac{1}{1-\delta} \mathbb{E}_{\delta} Z+\frac{1+\delta}{1-\delta} \sum_{n=1}^{\infty} z_{n} C_{n} \frac{-2 \delta n}{1-\delta^{2}}\left(\frac{1-\delta^{2}}{4}\right)^{n} \\
& =\frac{2}{1-\delta^{2}} \mathbb{E}_{\delta} Z-\frac{2 \delta}{1-\delta^{2}} \mathbb{E}_{\delta}(N Z) .
\end{aligned}
$$

The formula (5.5) follows.

(ii): An immediate consequence of (5.5).

As an example, taking $Z=1$ yields $f(\delta)=1$, and thus (5.5) yields

$$
\mathbb{E}_{\delta} N=\delta^{-1} \text {. }
$$

Taking $Z=N$ in (5.5) now yields

$$
\mathbb{E}_{\delta} N^{2}=\frac{1}{2} \delta^{-3}+\delta^{-2}-\frac{1}{2} \delta^{-1},
$$

and we can continue and find explicit expressions for $\mathbb{E}_{\delta} N^{m}$ for any desired $m$. (One can check that Lemma 5.4 is correct but trivial in these cases.)

After these preliminaries, we now consider the variables $X_{\sigma}$, and begin with their expectations for $T_{\delta}$. Recall that $\lambda(\sigma)$ is defined by (2.1).

Lemma 5.6. Let $\sigma \in \mathfrak{S}_{k}(132)$ with $k=|\sigma| \geqslant 1$ and define $m$ and $\Delta$ as in Lemma 3.2. Then $\mathbb{E}_{\delta} X_{\sigma}$ is a polynomial in $\delta^{-1}$ of degree $\lambda(\sigma)-1$ given by the recursion $\mathbb{E}_{\delta} X_{1}=\delta^{-1}$ and, for $k>1$,

$$
\begin{aligned}
\mathbb{E}_{\delta} X_{\sigma}=\delta^{-1} & \frac{(1-\delta)^{2}}{4} \sum_{q \in \Delta} \mathbb{E}_{\delta} X_{\sigma_{1} \cdots \sigma_{q}} \mathbb{E}_{\delta} X_{\sigma_{q+1} \cdots \sigma_{k}} \\
+ & \begin{cases}\frac{1}{2}\left(\delta^{-1}-1\right) \mathbb{E}_{\delta} X_{\sigma_{2} \cdots \sigma_{k}}, & m=1, \\
\frac{1}{4} \delta^{-1}(1-\delta)^{2} \mathbb{E}_{\delta} X_{\sigma_{1} \cdots \sigma_{m-1}} \mathbb{E}_{\delta} X_{\sigma_{m+1} \cdots \sigma_{k}}, & 1<m<k, \\
\frac{1}{2}\left(\delta^{-1}-1\right) \mathbb{E}_{\delta} X_{\sigma_{1} \cdots \sigma_{k-1}}, & m=k .\end{cases}
\end{aligned}
$$

The polynomial $\mathbb{E}_{\delta} X_{\sigma}$ has leading term $B_{\sigma} \delta^{-(\lambda(\sigma)-1)}$ and vanishing constant term, where $B_{\sigma}>0$ satisfies the recursion $B_{1}=1$ and, for $k>1$,

$$
B_{\sigma}=\frac{1}{4} \sum_{q \in \Delta} B_{\sigma_{1} \cdots \sigma_{q}} B_{\sigma_{q+1} \cdots \sigma_{k}}+ \begin{cases}\frac{1}{2} B_{\sigma_{1} \cdots \sigma_{k-1}}, & m=k \\ 0, & m<k\end{cases}
$$


Proof. We use induction on $\lambda(\sigma)$. We use the recursion in Lemma 4.1 and take expectations, considering the terms on the right-hand side of (4.1) separately.

Since $T_{L}$ is a copy of $T=T_{\delta}$ with probability $p=(1-\delta) / 2$ and empty with probability $1-p=(1-\delta) / 2$, and the same holds for $T_{R}$, we have

$$
\mathbb{E}_{\delta} X_{\sigma, L}=\mathbb{E}_{\delta} X_{\sigma, R}=p \mathbb{E}_{\delta} X_{\sigma}=\frac{1-\delta}{2} \mathbb{E}_{\delta} X_{\sigma} .
$$

Furthermore, $T_{L}$ and $T_{R}$ are independent, and thus, for $q \in \Delta$,

$$
\begin{aligned}
\mathbb{E}_{\delta}\left(X_{\sigma_{1} \cdots \sigma_{q}, L} X_{\sigma_{q+1} \cdots \sigma_{k}, R}\right) & =\mathbb{E}_{\delta}\left(X_{\sigma_{1} \cdots \sigma_{q}, L}\right) \mathbb{E}_{\delta}\left(X_{\sigma_{q+1} \cdots \sigma_{k}, R}\right) \\
& =\left(\frac{1-\delta}{2}\right)^{2} \mathbb{E}_{\delta}\left(X_{\sigma_{1} \cdots \sigma_{q}}\right) \mathbb{E}_{\delta}\left(X_{\sigma_{q+1} \cdots \sigma_{k}}\right) .
\end{aligned}
$$

By the induction hypothesis, this is a polynomial in $\delta^{-1}$ of degree

$$
\begin{aligned}
\lambda\left(\sigma_{1}\right. & \left.\cdots \sigma_{q}\right)-1+\lambda\left(\sigma_{q+1} \cdots \sigma_{k}\right)-1 \\
& =q+D\left(\sigma_{1} \cdots \sigma_{q}\right)-1+k-q+D\left(\sigma_{q+1} \cdots \sigma_{k}\right)-1 \\
& =k+D\left(\sigma_{1} \cdots \sigma_{k}\right)-2=\lambda(\sigma)-2,
\end{aligned}
$$

recalling that $q \in \Delta$ implies that $q$ is a descent in $\sigma$, which implies $D\left(\sigma_{1} \cdots \sigma_{q}\right)+$ $D\left(\sigma_{q+1} \cdots \sigma_{k}\right)=D\left(\sigma_{1} \cdots \sigma_{k}\right)$ by our definition of $D$. (Note that the induction assumption that the expectations are polynomials with vanishing constant term is used to guarantee that the right hand side of (5.10) is a polynomial in $\delta^{-1}$, even though it contains the factor $(1-\delta)^{2}$; the same applies below.)

For the final term in (4.1), we consider four different cases. First, if $1<m<k$, then as in (5.10)

$$
\mathbb{E}_{\delta}\left(X_{\sigma_{1} \cdots \sigma_{m-1}, L} X_{\sigma_{m+1} \cdots \sigma_{k}, R}\right)=\left(\frac{1-\delta}{2}\right)^{2} \mathbb{E}_{\delta}\left(X_{\sigma_{1} \cdots \sigma_{m-1}}\right) \mathbb{E}_{\delta}\left(X_{\sigma_{m+1} \cdots \sigma_{k}}\right),
$$

and this is a polynomial in $\delta^{-1}$ of degree

$$
\begin{aligned}
\lambda\left(\sigma_{1}\right. & \left.\cdots \sigma_{m-1}\right)-1+\lambda\left(\sigma_{m+1} \cdots \sigma_{k}\right)-1 \\
& =m-1+D\left(\sigma_{1} \cdots \sigma_{m-1}\right)-1+k-m+D\left(\sigma_{m+1} \cdots \sigma_{k}\right)-1 \\
& =\lambda(\sigma)-3 .
\end{aligned}
$$

If $m=1<k$, then the final term of (4.1) is simply $X_{\sigma_{2} \cdots \sigma_{k}, R}$, with an expectation that by induction is a polynomial in $\delta^{-1}$ of degree

$$
\lambda\left(\sigma_{2} \cdots \sigma_{k}\right)-1=k-1+D\left(\sigma_{2} \cdots \sigma_{k}\right)-1=\lambda(\sigma)-3,
$$

since 1 is a descent.

If $m=k>1$, then the final term of (4.1) is similarly $X_{\sigma_{1} \cdots \sigma_{k-1}, L}$, with an expectation that by induction is a polynomial in $\delta^{-1}$ of degree

$$
\lambda\left(\sigma_{1} \cdots \sigma_{k-1}\right)-1=k-1+D\left(\sigma_{1} \cdots \sigma_{k-1}\right)-1=\lambda(\sigma)-2,
$$

since $k-1$ is not a descent in $\sigma$. 
Finally, if $m=k=1$, i.e., if $\sigma=1$, the final term is simply 1 , again a polynomial of degree $\lambda(\sigma)-2$.

Collecting the terms above, we thus obtain from (4.1)

$$
\mathbb{E}_{\delta} X_{\sigma}=2 p \mathbb{E}_{\delta} X_{\sigma}+f(\delta)=(1-\delta) \mathbb{E}_{\delta} X_{\sigma}+f(\delta),
$$

where $f(\delta)$ is shorthand for a polynomial in $\delta^{-1}$ of degree (at most) $\lambda(\sigma)-2$, which yields

$$
\mathbb{E}_{\delta} X_{\sigma}=\delta^{-1} f(\delta)
$$

a polynomial in $\delta^{-1}$ of degree (at most) $\lambda(\sigma)-1$ and without constant term. Writing $f(\delta)$ explicitly, this yields the recursion stated in the lemma. For $\sigma=1$ we have $f(\delta)=1$ and (5.17) yields $\mathbb{E}_{\delta} X_{1}=\delta^{-1}$, as was found in another way in (5.6).

Moreover, an inspection of the leading terms above shows that the leading coefficient of $f(\delta)$ is $B_{\sigma}$ given by (5.8) when $|\sigma|>1$, and $B_{1}=1$. Thus, by induction, $B_{\sigma}>0$. (Recall that $\Delta \neq \emptyset$ if $m<k$, so the right-hand side of (5.8) contains at least one non-zero term.)

This completes the induction step.

It is now easy to show (2.5).

Corollary 5.7. For every $\sigma \in \mathfrak{S}_{*}(132)$,

$$
\mathbb{E} n_{\sigma}\left(\boldsymbol{\pi}_{132, n}\right)=\mathbb{E} X_{\sigma}\left(T_{n}\right) \sim A_{\sigma} n^{\lambda(\sigma) / 2},
$$

where

$$
A_{\sigma}=\frac{\sqrt{\pi}}{\Gamma((\lambda(\sigma)-1) / 2)} B_{\sigma},
$$

with $B_{\sigma}$ given by the recursion (5.8).

Proof. Immediate from Lemmas 5.6 and 5.4, together with (4.10).

Example 5.8. For $|\sigma|=1$, we have $\mathbb{E}_{\delta} N=\mathbb{E}_{\delta} X_{1}=\delta^{-1}$, as stated in (5.6)).

For $|\sigma|=2$, we have two cases. For $X_{12}=Y$ we obtain, cf. (4.5),

$$
\mathbb{E}_{\delta} X_{12}=\frac{1}{2}\left(\delta^{-1}-1\right) \mathbb{E}_{\delta} X_{1}=\frac{1}{2} \delta^{-2}-\frac{1}{2} \delta^{-1},
$$

Similarly, by Lemma 5.6 (with $\Delta=\{1\}$ ) and a short calculation, or by (4.3), (5.6) -(5.7) and (5.20),

$$
\mathbb{E}_{\delta} X_{21}=\frac{1}{4} \delta^{-3}-\frac{1}{4} \delta^{-1},
$$

For $|\sigma|=3$, we obtain from Lemma 5.6, or similarly from the explicit recursions in Example 4.2, by simple calculations,

$$
\begin{aligned}
& \mathbb{E}_{\delta} X_{123}=\frac{1}{4} \delta^{-3}-\frac{1}{2} \delta^{-2}+\frac{1}{4} \delta^{-1}, \\
& \mathbb{E}_{\delta} X_{213}=\frac{1}{8} \delta^{-4}-\frac{1}{8} \delta^{-3}-\frac{1}{8} \delta^{-2}+\frac{1}{8} \delta^{-1}, \\
& \mathbb{E}_{\delta} X_{231}=\frac{1}{8} \delta^{-4}-\frac{1}{8} \delta^{-3}-\frac{1}{8} \delta^{-2}+\frac{1}{8} \delta^{-1}, \\
& \mathbb{E}_{\delta} X_{312}=\frac{1}{8} \delta^{-4}-\frac{1}{8} \delta^{-3}-\frac{1}{8} \delta^{-2}+\frac{1}{8} \delta^{-1}, \\
& \mathbb{E}_{\delta} X_{321}=\frac{1}{8} \delta^{-5}-\frac{1}{8} \delta^{-4}-\frac{1}{8} \delta^{-3}+\frac{1}{8} \delta^{-2} .
\end{aligned}
$$


Note that $\mathbb{E}_{\delta} X_{213}=\mathbb{E}_{\delta} X_{231}=\mathbb{E}_{\delta} X_{312}$, which by Lemma 5.2 is equivalent to the result by Bóna [12] $\mathbb{E} n_{213}\left(\boldsymbol{\pi}_{132, n}\right)=\mathbb{E} n_{231}\left(\boldsymbol{\pi}_{132, n}\right)=\mathbb{E} n_{312}\left(\boldsymbol{\pi}_{132, n}\right)$, as mentioned earlier in (1.5).

The asymptotics (1.3)-(1.6) follow from Corollary 5.7 and (5.8). Alternatively, we can obtain these from the explicit formulas (5.20)-(5.26) and Lemma 5.4

Remark 5.9. When $Z=X_{\sigma}=n_{\sigma}\left(\boldsymbol{\pi}_{132, n}\right), z_{n}$ is the expected number of occurrences of $\sigma$ in a random permutation in $\mathfrak{S}_{n}(132)$, and $C_{n} z_{n}$ is thus the total number of occurrences of $\sigma$ in all permutations in $\mathfrak{S}_{n}(132)$. Generating functions for the latter numbers have been given for the cases in Example 5.8 (although not explicitly for 321) by Bóna [10] and [12]; by Lemma 5.2 and Remark 5.3, the formulas $(5.20)-(5.25)$ are equivalent to his results.

Remark 5.10. As said in Section 1, $n_{231}\left(\boldsymbol{\pi}_{132, n}\right)$ and $n_{312}\left(\boldsymbol{\pi}_{132, n}\right)$ have the same distribution by symmetry, and thus $\mathbb{E}_{\delta} X_{231}=\mathbb{E}_{\delta} X_{312}$ is obvious. It is interesting that the proof above obtains these coinciding expectations by different routes, using the different recursions (4.8) and (4.9). The same applies to the higher moments treated below: $\mathbb{E}_{\delta} X_{231}^{k}=\mathbb{E}_{\delta} X_{312}^{k}$ for any $k$, but that is difficult to see from our recursions.

Example 5.11. For $|\delta|=4$, there are $C_{4}=14$ permutations $\sigma \in \mathfrak{S}_{4}$. Lemma 5.6 yields the following formulas.

$$
\begin{aligned}
\mathbb{E}_{\delta} X_{1234} & =\frac{1}{8} \delta^{-4}-\frac{3}{8} \delta^{-3}+\frac{3}{8} \delta^{-2}-\frac{1}{8} \delta^{-1} \\
\mathbb{E}_{\delta} X_{2134} & =\mathbb{E}_{\delta} X_{2314}=\mathbb{E}_{\delta} X_{2341}=\mathbb{E}_{\delta} X_{3124}=\mathbb{E}_{\delta} X_{3412}=\mathbb{E}_{\delta} X_{4123} \\
& =\frac{1}{16} \delta^{-5}-\frac{1}{8} \delta^{-4}+\frac{1}{8} \delta^{-2}-\frac{1}{16} \delta^{-1} \\
\mathbb{E}_{\delta} X_{3214} & =\mathbb{E}_{\delta} X_{3421}=\mathbb{E}_{\delta} X_{4231}=\mathbb{E}_{\delta} X_{4312} \\
& =\frac{1}{16} \delta^{-6}-\frac{1}{8} \delta^{-5}+\frac{1}{8} \delta^{-3}-\frac{1}{16} \delta^{-2} \\
\mathbb{E}_{\delta} X_{3241} & =\mathbb{E}_{\delta} X_{4213} \\
& =\frac{1}{32} \delta^{-6}-\frac{1}{32} \delta^{-5}-\frac{1}{16} \delta^{-4}+\frac{1}{16} \delta^{-3}+\frac{1}{32} \delta^{-2}-\frac{1}{32} \delta^{-1} \\
\mathbb{E}_{\delta} X_{4321} & =\frac{5}{64} \delta^{-7}-\frac{5}{32} \delta^{-6}-\frac{1}{64} \delta^{-5}+\frac{3}{16} \delta^{-4}-\frac{5}{64} \delta^{-3}-\frac{1}{32} \delta^{-2}+\frac{1}{64} \delta^{-1}
\end{aligned}
$$

We see again several coincidences, which by Lemma 5.2 imply corresponding equalities for each $n$, for a random 132-avoiding permutation $\boldsymbol{\pi}_{132, n}$ :

$$
\begin{aligned}
& \mathbb{E} n_{2134}=\mathbb{E} n_{2314}=\mathbb{E} n_{2341}=\mathbb{E} n_{3124}=\mathbb{E} n_{3412}=\mathbb{E} n_{4123}, \\
& \mathbb{E} n_{3214}=\mathbb{E} n_{3421}=\mathbb{E} n_{4231}=\mathbb{E} n_{4312}, \\
& \mathbb{E} n_{3241}=\mathbb{E} n_{4213} .
\end{aligned}
$$

Some equalities are obvious by the inversion symmetry in Remark 1.1, others follow by Bóna [12] and all are contained in the result by Rudolph [39].

Asymptotic results follow by (5.27)-(5.31) and Lemma 5.4, or directly by Corollary 5.7 and (5.8); we leave these to the reader. It is also possible to 
obtain exact expressions for finite $n$ by (5.4) and Taylor expansion; we leave these too to the reader.

Example 5.12. For $\sigma=1 \cdots k, k \geqslant 1$, we have $\Delta=\emptyset$ and Lemma 5.6 yields by induction in $k$

$$
\mathbb{E}_{\delta} X_{1 \cdots k}=2^{1-k}\left(\delta^{-1}-1\right)^{k-1} \delta^{-1} .
$$

This is by Lemma 5.2 and Remark 5.3 equivalent to the generating function given for this case by Bóna [10].

Lemma 5.4 and (5.35) yield (1.7).

Example 5.13. For $\sigma=k \cdots 1, k \geqslant 1$, we have the opposite extreme $\Delta=$ $[k-1]$. Lemma 5.6 yields the recursion, where we write $f_{k}(\delta)=\mathbb{E}_{\delta} X_{k \cdots 1}$,

$$
f_{k}(\delta)=\frac{1}{4} \delta^{-1}(1-\delta)^{2} \sum_{q=1}^{k-1} f_{q}(\delta) f_{k-q}(\delta)+\frac{1}{2}\left(\delta^{-1}-1\right) f_{k-1}(\delta),
$$

which by (5.4) is equivalent to the recursion given for the corresponding generating functions in Bóna [10].

The leading term $B_{k \cdots 1} \delta^{-(2 k-1)}$ is given by the recursion (5.8), but it is simpler to argue backwards and note that $A_{k \cdots 1}=1 / k$ ! by Theorem 2.1 , see also Remark 2.4, and thus (5.19) yields

$$
B_{k \cdots 1}=\frac{\Gamma(k-1 / 2)}{\Gamma(1 / 2) k !}=\frac{(2 k-3) ! !}{2^{k-1} k !}=\frac{C_{k-1}}{2^{2 k-2}} .
$$

See the examples in (5.21), (5.26), (5.31).

\section{HigheR MOMENTS}

We can compute higher moments in the same way.

Lemma 6.1. For any permutations $\sigma^{(1)}, \ldots, \sigma^{(\nu)} \in \mathfrak{S}_{*}(132)$, not necessarily distinct, $\mathbb{E}_{\delta}\left(X_{\sigma^{(1)}} \cdots X_{\sigma^{(\nu)}}\right)$ is a polynomial in $\delta^{-1}$ of degree $\sum_{j=1}^{\nu} \lambda\left(\sigma^{(j)}\right)-1$, with positive leading coefficient $B_{\sigma^{(1)}, \ldots, \sigma^{(\nu)}}$ and vanishing constant term.

Proof. We argue as in the proof of Lemma 5.6, using induction on $\sum_{j=1}^{\nu} \lambda\left(\sigma^{(j)}\right)$. Replace each $X_{\sigma^{(j)}}$ by the corresponding expression in (4.1), expand the product of these, and take the expectation. Among the many terms that this produces, the two special ones $\mathbb{E}_{\delta}\left(X_{\sigma^{(1)}, L} \cdots X_{\sigma^{(\nu)}, L}\right)$ and $\mathbb{E}_{\delta}\left(X_{\sigma^{(1)}, R} \cdots X_{\sigma^{(\nu)}, R}\right)$ are both equal to $p \mathbb{E}_{\delta}\left(X_{\sigma^{(1)}} \cdots X_{\sigma^{(\nu)}}\right)$. All other terms are by induction polynomials in $\delta^{-1}$, of degrees at most $\sum_{j=1}^{\nu} \lambda\left(\sigma^{(j)}\right)-2$ (by arguing similarly to the proof of Lemma 5.6 for each $\sigma^{(j)}$ ); moreover, there is at least one term of exactly this degree and all polynomials have positive leading coefficients. The result follows as in Lemma 5.6. 
Example 6.2. By squaring (4.5) and taking the expectation we obtain

$$
\begin{aligned}
\mathbb{E}_{\delta} Y^{2} & =\mathbb{E}_{\delta}\left(Y_{L}+N_{L}\right)^{2}+\mathbb{E}_{\delta} Y_{R}^{2}+2 \mathbb{E}_{\delta}\left(Y_{L}+N_{L}\right) \mathbb{E}_{\delta} Y_{R} \\
& =p \mathbb{E}_{\delta}(Y+N)^{2}+p \mathbb{E}_{\delta} Y^{2}+2 p^{2} \mathbb{E}_{\delta}(Y+N) \mathbb{E}_{\delta} Y \\
& =2 p \mathbb{E}_{\delta} Y^{2}+2 p \mathbb{E}_{\delta}(N Y)+p \mathbb{E}_{\delta} N^{2}+2 p^{2}\left(\mathbb{E}_{\delta} Y\right)^{2}+2 p^{2} \mathbb{E}_{\delta} Y \mathbb{E}_{\delta} N .
\end{aligned}
$$

Hence, recalling $2 p=1-\delta$,

$$
\mathbb{E}_{\delta} Y^{2}=\delta^{-1}\left(2 p \mathbb{E}_{\delta}(N Y)+p \mathbb{E}_{\delta} N^{2}+2 p^{2}\left(\mathbb{E}_{\delta} Y\right)^{2}+2 p^{2} \mathbb{E}_{\delta} Y \mathbb{E}_{\delta} N\right),
$$

which can be written as an explicit polynomial in $\delta^{-1}$ by (5.6) $-(5.7),(5.20)$ and Lemma 5.5. Using this, we then can find, for example, $\mathbb{E}\left(X_{123} Y\right)$ by multiplying (4.5) and (4.6) and taking the expectation, and then $\mathbb{E} X_{123}^{2}$ by squaring (4.6) and using the same argument again.

In this way we can recursively obtain any mixed moment of the variables $X_{\sigma}$ as a polynomial in $\delta^{-1}$. For simplicity, we leave exact formulas to the reader, and consider only the leading terms, which by Lemma 5.4 will yield the moment asymptotics for $T_{n}$ that we desire.

A recursion for the leading coefficients $B_{\sigma^{(1)}, \ldots, \sigma^{(\nu)}}$ is implicit in the proof above, but to write it explicitly in general seems a bit messy, so we restrict ourselves in the rest of this section to the case $|\sigma| \leqslant 3$, which gives examples illustrating the general behaviour.

We consider first a single $X_{\sigma}$ with $|\sigma|=3$, but for the induction, we have to consider mixed moments of $X_{\sigma}$ and $Y=X_{12}$.

\section{Lemma 6.3.}

(i) If $k \geqslant 0$ and $l \geqslant 0$ with $k+l \geqslant 1$, then

$$
\mathbb{E}_{\delta}\left(X_{123}^{k} Y^{l}\right)=a_{k l} \delta^{-(4 k+3 l-1)}+O\left(\delta^{-(4 k+3 l-2)}\right)
$$

for some positive numbers $a_{k l}$ satisfying $a_{01}=\frac{1}{2}, a_{10}=\frac{1}{4}$ and the recursion relation

$$
a_{k, l}=\frac{k}{2} a_{k-1, l+1}+\frac{l(4 k+3 l-4)}{4} a_{k, l-1}+\frac{1}{4} \sum_{0<i+j<k+l} \sum_{0}\left(\begin{array}{l}
k \\
i
\end{array}\right)\left(\begin{array}{l}
l \\
j
\end{array}\right) a_{i, j} a_{k-i, l-j} .
$$

(ii) If $k \geqslant 0$ and $l \geqslant 0$ with $k+l \geqslant 1$, then

$$
\mathbb{E}_{\delta}\left(X_{213}^{k} Y^{l}\right)=b_{k l} \delta^{-(5 k+3 l-1)}+O\left(\delta^{-(5 k+3 l-2)}\right)
$$

for some positive numbers $b_{k l}$ satisfying $b_{01}=\frac{1}{2}, b_{10}=\frac{1}{8}$ and the recursion relation

$$
\begin{aligned}
b_{k, l}=\frac{k(5 k+3 l-6)(5 k+3 l-4)}{16} & b_{k-1, l}+\frac{l(5 k+3 l-4)}{4} b_{k, l-1} \\
+ & \frac{1}{4} \sum_{0<i+j<k+l} \sum_{i}\left(\begin{array}{l}
k \\
i
\end{array}\right)\left(\begin{array}{l}
l \\
j
\end{array}\right) b_{i, j} b_{k-i, l-j} .
\end{aligned}
$$


(iii) If $k \geqslant 0$ and $l \geqslant 0$ with $k+l \geqslant 1$, then

$$
\mathbb{E}_{\delta}\left(X_{231}^{k} Y^{l}\right)=\mathbb{E}_{\delta}\left(X_{312}^{k} Y^{l}\right)=c_{k l} \delta^{-(5 k+3 l-1)}+O\left(\delta^{-(5 k+3 l-2)}\right)
$$

for some positive numbers $c_{k l}$ satisfying $c_{01}=\frac{1}{2}, c_{10}=\frac{1}{8}$ and the recursion relation

$$
\begin{array}{r}
c_{k, l}=\frac{l(5 k+3 l-4)}{4} c_{k, l-1}+\frac{1}{4} \sum_{(i, j, m) \neq(0,0,0),(k, l, 0)}\left(\begin{array}{c}
k \\
i, m, k-i-m
\end{array}\right)\left(\begin{array}{l}
l \\
j
\end{array}\right) \times \\
\frac{\Gamma((5 i+3 j-1) / 2+m))}{\Gamma((5 i+3 j-1) / 2))} c_{i, j} c_{k-i-m, l-j+m} .
\end{array}
$$

Proof. (i) Note that (5.20) and (5.22) show that (6.1) holds when $k+l=1$, with $a_{01}=\frac{1}{2}, a_{10}=\frac{1}{4}$. We continue by induction, and assume that $K, L \geqslant 0$ with $K+L \geqslant 2$ are such that (6.1) holds when $1 \leqslant 4 k+3 l<4 K+3 L$. For such $k$ and $l$ and any $m \geqslant 0$, Lemma 5.5(ii) implies

$$
\begin{aligned}
\mathbb{E}_{\delta}\left(X_{123}^{k} Y^{l} N^{m}\right)=a_{k l} \prod_{j=0}^{m-1} \frac{4 k+3 l+2 j-1}{2} & \delta^{-(4 k+3 l+2 m-1)} \\
& +O\left(\delta^{-(4 k+3 l+2 m-2)}\right) \\
= & O\left(\delta^{-(4 k+3 l+2 m-1)}\right) .
\end{aligned}
$$

The same holds for $k=l=0$ and $m \geqslant 1$ too (with $a_{00}=-2$ ) by (5.6) and Lemma 5.5 .

Now consider $k=K$ and $l=L$. By (4.6), (4.5) and the binomial theorem,

$$
\begin{aligned}
\mathbb{E}_{\delta}\left(X_{123}^{k} Y^{l}\right)=\sum_{k_{1}+k_{2}+k_{3}=k} \sum_{l_{1}+l_{2}+l_{3}=l}\left(\begin{array}{c}
k \\
k_{1}, k_{2}, k_{3}
\end{array}\right)\left(\begin{array}{c}
l \\
l_{1}, l_{2}, l_{3}
\end{array}\right) \times \\
\mathbb{E}_{\delta}\left(X_{123, L}^{k_{1}} X_{123, R}^{k_{2}} Y_{L}^{k_{3}+l_{1}} Y_{R}^{l_{2}} N_{L}^{l_{3}}\right) .
\end{aligned}
$$

Consider one of the terms in the sum. If this term contains both $L$-factors and $R$-factors, i.e., if $k_{1}+k_{3}+l_{1}+l_{3}>0$ and $k_{2}+l_{2}>0$, then the expectation is, by the induction hypothesis and (6.7),

$$
p^{2} \mathbb{E}_{\delta}\left(X_{123}^{k_{1}} Y^{k_{3}+l_{1}} N^{l_{3}}\right) \mathbb{E}_{\delta}\left(X_{123}^{k_{2}} Y^{l_{2}}\right)=O\left(\delta^{-\left(4 k_{1}+3 k_{3}+3 l_{1}+2 l_{3}+4 k_{2}+3 l_{2}-2\right)}\right)
$$

If $k_{3}>0$ or $l_{3}>0$, this term is of lower order than $\delta^{-(4 k+3 l-2)}$, and we see, using the induction hypothesis again, that the sum of the terms in (6.8) with both $L$-factors and $R$-factors is

$$
\sum_{0<k_{1}+l_{1}<k+l} \sum_{k_{1}}\left(\begin{array}{c}
k \\
k_{1}
\end{array}\right)\left(\begin{array}{l}
l \\
l_{1}
\end{array}\right) p^{2} a_{k_{1}, l_{1}} a_{k-k_{1}, l-l_{1}} \delta^{-(4 k+3 l-2)}+O\left(\delta^{-(4 k+3 l-3)}\right) .
$$

The terms in (6.8) with only $L$-factors are the ones with $k_{2}=l_{2}=0$. The induction hypothesis and (6.7) now show that the term is of order 
$O\left(\delta^{-4 k_{1}+4 k_{3}+3 l_{1}+2 l_{3}-1}\right)$, and thus only terms with $k_{3}+l_{3} \leqslant 1$ are significant. The sum of these terms is thus, using (6.7),

$$
\begin{aligned}
& p \mathbb{E}_{\delta}\left(X_{123}^{k} Y^{l}\right)+p k \mathbb{E}_{\delta}\left(X_{123}^{k-1} Y^{l+1}\right)+p l \mathbb{E}_{\delta}\left(X_{123}^{k} Y^{l-1} N\right)+O\left(\delta^{-(4 k+3 l-3)}\right) \\
&=p \mathbb{E}_{\delta}\left(X_{123}^{k} Y^{l}\right)+p k a_{k-1, l+1} \delta^{-(4 k+3 l-2)} \\
&+p l a_{k, l-1} \frac{4 k+3 l-4}{2} \delta^{-(4 k+3 l-2)}+O\left(\delta^{-(4 k+3 l-3)}\right) .
\end{aligned}
$$

Finally, the only term in (6.8) with only $R$-factors is

$$
\mathbb{E}_{\delta}\left(X_{123, R}^{k} Y_{R}^{l}\right)=p \mathbb{E}_{\delta}\left(X_{123}^{k} Y^{l}\right) .
$$

Using $p=(1-\delta) / 2$, we thus obtain by collecting the terms in (6.8),

$$
\begin{aligned}
& \delta \mathbb{E}_{\delta}\left(X_{123}^{k} Y^{l}\right)=\frac{1}{2} k a_{k-1, l+1} \delta^{-(4 k+3 l-2)}+\frac{1}{2} l a_{k, l-1} \frac{4 k+3 l-4}{2} \delta^{-(4 k+3 l-2)} \\
& +\sum_{0<k_{1}+l_{1}<k+l}\left(\begin{array}{c}
k \\
k_{1}
\end{array}\right)\left(\begin{array}{l}
l \\
l_{1}
\end{array}\right) \frac{1}{4} a_{k_{1}, l_{1}} a_{k-k_{1}, l-l_{1}} \delta^{-(4 k+3 l-2)}+O\left(\delta^{-(4 k+3 l-3)}\right),
\end{aligned}
$$

which completes the induction.

(ii) Similar, with $4 k$ replaced by $5 k$ and using (4.7); the main difference is that the significant terms with only $L$-factors now are $\mathbb{E}_{\delta}\left(X_{123, L}^{k} Y_{L}^{l}\right)$, $k \mathbb{E}_{\delta}\left(X_{123, L}^{k-1} Y_{L}^{l}\left(\begin{array}{c}N_{L} \\ 2\end{array}\right)\right)$ and $l \mathbb{E}_{\delta}\left(X_{123, L}^{k} Y_{L}^{l-1} N_{L}\right)$, where the first and third terms are as above and the second is handled by the analogue of (6.7).

(iii) The equality $\mathbb{E}_{\delta}\left(X_{231}^{k} Y^{l}\right)=\mathbb{E}_{\delta}\left(X_{312}^{k} Y^{l}\right)$ follows from the inversion symmetry in Remark 1.1, which implies that $\left(X_{231}, Y\right) \stackrel{\mathrm{d}}{=}\left(X_{312}, Y\right)$ by translating first to $T_{n}$ by (4.10) and then to $T_{\delta}$ by taking a random $n$. For the recursion we can use any of (4.8) and (4.9); the leading terms will be the same. The main difference in the induction is that (using (4.8)) the significant terms with both $L$-factors and $R$-factors now are all terms

$$
\begin{aligned}
\left(\begin{array}{c}
k \\
k_{1}, k_{2}, k_{3}
\end{array}\right)\left(\begin{array}{c}
l \\
l_{1}
\end{array}\right) & \mathbb{E}_{\delta}\left(X_{231, L}^{k_{1}} X_{231, R}^{k_{2}} N_{R}^{k_{3}} Y_{L}^{k_{3}+l_{1}} Y_{R}^{l_{2}}\right) \\
= & \left(\begin{array}{c}
k \\
k_{1}, k_{2}, k_{3}
\end{array}\right)\left(\begin{array}{c}
l \\
l_{1}
\end{array}\right) p^{2} \mathbb{E}_{\delta}\left(X_{231}^{k_{1}} Y^{k_{3}+l_{1}}\right) \mathbb{E}_{\delta}\left(X_{231}^{k_{2}} Y^{l_{2}} N^{k_{3}}\right),
\end{aligned}
$$

except the terms with $k_{1}+k_{3}+l_{1}=0$ or $k_{2}+k_{3}+l_{2}=0$, which, using the analogue of (6.7), leads to the recursion (6.6). (We write $i=k_{2}, j=l_{2}$, $m=k_{3}$.)

Remark 6.4. The proof (or a direct inspection) shows that the recursions (6.2), (6.4), (6.6) hold also for $k+l=1$, provided we define $a_{0,0}=b_{0,0}=$ $c_{0,0}:=-2$.

This yields the moment asymptotics.

Theorem 6.5. The following hold as $n \rightarrow \infty$, for any integers $k \geqslant 0$ and $l \geqslant 0$. 
(i)

$$
n^{-(4 k+3 l) / 2} \mathbb{E}\left(X_{123}\left(T_{n}\right)^{k} Y\left(T_{n}\right)^{l}\right) \rightarrow \frac{k ! l ! \sqrt{\pi}}{2^{4 k+3 l-2} \Gamma((4 k+3 l-1) / 2)} \alpha_{k l}
$$

for some numbers $\alpha_{k l}$ satisfying $\alpha_{0,0}=-1 / 2, \alpha_{10}=\alpha_{01}=1$ and the recursion relation

$$
\alpha_{k, l}=(l+1) \alpha_{k-1, l+1}+2(4 k+3 l-4) \alpha_{k, l-1}+\sum_{0<i+j<k+l} \sum_{i, j} \alpha_{k-i, l-j} .
$$

$$
n^{-(5 k+3 l) / 2} \mathbb{E}\left(X_{213}\left(T_{n}\right)^{k} Y\left(T_{n}\right)^{l}\right) \rightarrow \frac{k ! l ! \sqrt{\pi}}{2^{5 k+3 l-2} \Gamma((5 k+3 l-1) / 2)} \beta_{k l}
$$

for some numbers $\beta_{k l}$ satisfying $\beta_{0,0}=-1 / 2, \beta_{10}=\beta_{01}=1$ and the recursion relation

$$
\begin{aligned}
\beta_{k, l}=2(5 k+3 l-6)(5 k+3 l-4) \beta_{k-1, l}+ & 2(5 k+3 l-4) \beta_{k, l-1} \\
& +\sum_{0<i+j<k+l} \sum_{i, j} \beta_{k-i, l-j} .
\end{aligned}
$$

$$
\begin{aligned}
n^{-(5 k+3 l) / 2} \mathbb{E}\left(X_{231}\left(T_{n}\right)^{k} Y\left(T_{n}\right)^{l}\right) & =n^{-(5 k+3 l) / 2} \mathbb{E}\left(X_{312}\left(T_{n}\right)^{k} Y\left(T_{n}\right)^{l}\right) \\
& \rightarrow \frac{k ! l ! \sqrt{\pi}}{2^{5 k+3 l-2} \Gamma((5 k+3 l-1) / 2)} \gamma_{k l}
\end{aligned}
$$

for some numbers $\gamma_{k l}$ satisfying $\gamma_{0,0}=-1 / 2, \gamma_{10}=\gamma_{01}=1$ and the recursion relation

$$
\begin{aligned}
\gamma_{k, l}= & 2(5 k+3 l-4) \gamma_{k, l-1}+\sum_{(i, j, m) \neq(0,0,0),(k, l, 0)} \sum \\
& 2^{2 m} \frac{\Gamma((5 i+3 j-1) / 2+m))}{\Gamma((5 i+3 j-1) / 2))}\left(\begin{array}{c}
l-j+m \\
m
\end{array}\right) \gamma_{i, j} \gamma_{k-i-m, l-j+m} .
\end{aligned}
$$

Proof. Immediate from Lemmas 6.3 and 5.4 with the definitions

$$
\begin{aligned}
\alpha_{k, l} & :=\frac{2^{4 k+3 l-2}}{k ! l !} a_{k, l}, \\
\beta_{k, l} & :=\frac{2^{5 k+3 l-2}}{k ! l !} b_{k, l}, \\
\gamma_{k, l} & :=\frac{2^{5 k+3 l-2}}{k ! l !} c_{k, l} .
\end{aligned}
$$

The choice $\alpha_{0,0}=\beta_{0,0}=\gamma_{0,0}:=-1 / 2$ satisfies both (6.10), (6.12), (6.14) for $k=l=0$ (trivially) and the recursions (6.11), (6.13), 6.15) for $k+l=1$, cf. Remark 6.4,

Note that when we have proved Theorem 2.1, it follows that the limits in (6.10), (6.12), (6.14) are equal to the moments $\mathbb{E}\left(\Lambda_{123}^{k} \Lambda_{12}^{l}\right)$, etc. 
Remark 6.6. The number $\beta_{k, l}$ in Theorem 6.5)(ii) satisfy the same recursion as $\omega_{l, k}^{*}$ in [23], and thus $\beta_{k, l}=\omega_{l, k}^{*}$. Indeed they both appear in similar moment formulas, and the equality is explained by the identities in Remark 7.12 below.

In the same way it is possible to find mixed moments of these variables, first for $T_{\delta}$ and then (asymptotically, or exact) for $T_{n}$. We give only an example.

Example 6.7. Let $\left(V_{1}, V_{2}, V_{3}\right)=\left(X_{213}\left(T_{n}\right), X_{231}\left(T_{n}\right), X_{312}\left(T_{n}\right)\right)$ be the three random variables in (1.5); recall that these have equal mean. Using the recursions (4.7)-(4.9), the method in the proof of Lemma 6.1 yields $\mathbb{E}_{\delta}\left(V_{i} V_{j}\right)$ as polynomials in $\delta^{-1}$ of degree 9 . After calculating the leading coefficients (we omit the details), we obtain from Lemma 5.4, in matrix notation,

$$
\left(n^{-5} \mathbb{E}\left(V_{i} V_{j}\right)\right)_{i, j=1}^{3} \rightarrow \frac{1}{840}\left(\begin{array}{ccc}
49 & 42 & 42 \\
42 & 43 & 41 \\
42 & 41 & 43
\end{array}\right)
$$

\section{BROWNIAN FUNCTIONALS}

Given a binary tree $T$, let $h(v)=h(v ; T)$ be the height (also called depth) of a vertex $v \in T$, defined as the distance to the root. Thus $h(v)$ is the number of ancestors of $v$. We define also the left height $h_{L}(v)$ as the number of ancestors $w$ of $v$ such that $v$ belongs to the left subtree of $w$, and similarly the right height $h_{R}(v)$. Equivalently, $h_{L}(v)$ is the number of left steps in the path to $v$.

Define the profile of a binary tree $T$ as the sequence $h\left(v_{1}\right), \ldots, h\left(v_{n}\right)$, where $v_{1}, \ldots, v_{n}$ are the vertices of $T$ in inorder; recall that the inorder is defined recursively by taking first the vertices of $T_{L}$, then the root and then the vertices of $T_{R}$ [27, Section 2.3.1]. We write $h(i)=h\left(v_{i}\right)$ and regard $h$ as a function both on the vertex set of $T$ and on $[n]$. We further define, for $1 \leqslant i \leqslant j \leqslant n$,

$$
h([i, j]):=\min _{l \in[i, j]} h(l) .
$$

It is well known that for the random binary tree $T_{n}$, the height $h(v)$ is typically of the order $n^{1 / 2}$. For example, if $H\left(T_{n}\right):=\max _{v \in T_{n}} h(v)$ is the height of $T_{n}$, then $H\left(T_{n}\right) / n^{1 / 2}$ converges in distribution as $n \rightarrow \infty$ (e.g. as a consequence of Lemma 7.1 below, see [3]). Moreover, if we normalize the profile by defining

$$
\tilde{h}(x)=\tilde{h}\left(x ; T_{n}\right):=n^{-1 / 2} h\left(\lfloor n x\rfloor+1 ; T_{n}\right)
$$

(with $\tilde{h}(1)=0$ ), which is a function $[0,1] \rightarrow[0, \infty)$, then the random function $\tilde{h}\left(x ; T_{n}\right)$ converges in distribution to the standard normalized Brownian excusion $\mathbf{e}(x)$, up to a constant factor, as stated in the following lemma, 
in principle due to Aldous [4]. (Informally, e can be seen as Brownian motion on $[0,1]$ conditioned on $\mathbf{e}(x) \geqslant 0$ and $\mathbf{e}(1)=\mathbf{e}(0)=0$. For formal treatments, see e.g. [18] and [36].)

Lemma 7.1. As $n \rightarrow \infty, \tilde{h}\left(x ; T_{n}\right) \stackrel{\mathrm{d}}{\longrightarrow} 2^{3 / 2} \mathbf{e}(x)$.

Remark 7.2. The convergence in Lemma 7.1 is in the space $D[0,1]$ of right-continous functions with left limits. (We could have defined $\tilde{h}$ as a continuous function instead, using linear interpolation of $h(i)$ between integers, with no other essential differences below, and then the convergence would have been in $C[0,1]$.) For a full technical discussion of convergence in distribution in $D[0,1]$ or $C[0,1]$, see e.g. 7]. For our purposes, we may avoid technicalities by the Skorohod representation theorem [26, Theorem 4.30], which shows that we may assume that the random trees $T_{n}$ for different $n$, and e, are coupled such that the conclusion $\tilde{h}\left(x ; T_{n}\right) \stackrel{\mathrm{d}}{\longrightarrow} 2^{3 / 2} \mathbf{e}(x)$ holds a.s., uniformly for $x \in[0,1]$, i.e., $\sup _{x \in[0,1]}\left|\tilde{h}\left(x ; T_{n}\right)-2^{3 / 2} \mathbf{e}(x)\right| \rightarrow 0$ a.s.

Proof. As said above, this is in principle due to Aldous [4]. More precisely, Aldous considered the depth first walk on $T_{n}$, which is the sequence of vertices $w_{0}, \ldots, w_{2 n-2}$ obtained by walking along the "outside of the tree", with $w_{0}=w_{2 n-2}=o$, the root, and beginning with the left subtree (if any), see e.g. [17, Section 4.1.1]. Define $f(i):=h\left(w_{i}\right)$ and the normalized version $\tilde{f}(x):=n^{-1 / 2} f(\lfloor 2 n x\rfloor)$ for $x \in[0,1]$ (with $f(2 n-1)=f(2 n)=0$ for completeness). Aldous [4, Theorem 23] proved (in greater generality) that then $\tilde{f}(x) \stackrel{\mathrm{d}}{\longrightarrow} 2^{3 / 2} \mathbf{e}(x)$.

Some variations (and a new proof) were given by Marckert and Mokkadem [34], including a version with process of heights of the vertices taken in depth first order (first the root, then $T_{L}$, then $T_{R}$ ). In the present paper we use instead the inorder, but the argument in [34] is easily adapted to this case too, as follows.

Consider a vertex $v$ in a binary tree $T$. Let $T_{L}(v)$ and $T_{R}(v)$ denote the left and right subtrees of $v$, and let $\mathcal{P}_{v}$ be the set of the ancestors of $v$ (i.e., the path from the root to $v$, except $v$ itself). It is easily seen that the vertices that come before $i$ in the inorder are (i) the set $\mathcal{P}_{v, R}:=\left\{w \in \mathcal{P}_{v}: v \in T_{R}(w)\right\}$ and (ii) $\mathcal{L}_{v}:=\bigcup_{w \in \mathcal{P}_{v, R} \cup\{v\}} T_{L}(w)$. Hence, $v=v_{i}$, where

$$
i=1+h_{R}(v)+\left|\mathcal{L}_{v}\right| \cdot
$$

Similarly, since it takes the depth first walk $2 m$ steps to visit a subtree of size $m$, it is easily seen that if

$$
j:=h(v)+2\left|\mathcal{L}_{v}\right|
$$

then $w_{j}=v=v_{i}$. Note that

$$
|2 i-j|=\left|2+2 h_{R}(v)-h(v)\right|=\left|2+h_{R}(v)-h_{L}(v)\right| \leqslant 2+H .
$$


Now consider again $T_{n}$. Let $x \in[0,1)$ and let $i:=\lfloor n x\rfloor+1$. Find the corresponding vertex $v_{i} \in T_{n}$ and define $j$ as above, and $y:=j /(2 n)$. Then

$$
\tilde{h}(x)=n^{-1 / 2} h\left(v_{i}\right)=n^{-1 / 2} h\left(w_{j}\right)=n^{-1 / 2} h\left(w_{2 n y}\right)=\tilde{f}(y)
$$

and, by (7.5),

$$
|x-y| \leqslant\left|x-\frac{i}{n}\right|+\frac{|2 i-j|}{2 n} \leqslant \frac{4+H}{2 n} .
$$

By the result by Aldous [4] and Remark [7.2, we may assume that $\sup \mid \tilde{f}(x)-$ $2^{3 / 2} \mathbf{e}(x) \mid \rightarrow 0$ a.s. as $n \rightarrow \infty$. By (7.6) and (7.7),

$$
\begin{aligned}
\left|\tilde{h}(x)-2^{3 / 2} \mathbf{e}(x)\right| & =\left|\tilde{f}(y)-2^{3 / 2} \mathbf{e}(x)\right| \\
& \leqslant\left|\tilde{f}(y)-2^{3 / 2} \mathbf{e}(y)\right|+2^{3 / 2}|\mathbf{e}(y)-\mathbf{e}(x)| \\
& \leqslant \sup _{y}\left|\tilde{f}(y)-2^{3 / 2} \mathbf{e}(y)\right|+2^{3 / 2} \sup _{|x-y| \leqslant(H+4) / 2 n}|\mathbf{e}(y)-\mathbf{e}(x)| .
\end{aligned}
$$

The right-hand side does not depend on $x$ and tends to 0 a.s., by the result of Aldous [4], its immediate consequence $H / n \rightarrow 0$, and the continuity of e.

Actually, we need the corresponding result for the left height $h_{L}$. We define, in analogy with (7.2),

$$
\tilde{h}_{L}(x)=\tilde{h}\left(l ; T_{n}\right):=n^{-1 / 2} h_{L}\left(\lfloor n x\rfloor+1 ; T_{n}\right) .
$$

The following version of Lemma 7.1 is in principle due to Marckert [33].

Lemma 7.3. As $n \rightarrow \infty, \tilde{h}_{L}\left(x ; T_{n}\right) \stackrel{\mathrm{d}}{\longrightarrow} 2^{1 / 2} \mathbf{e}(x)$.

Proof. Marckert [33] proved this for the depth first order; and, moreover, that

$$
n^{-1 / 2} \max _{v \in T_{n}}\left|h_{L}(v)-h_{R}(v)\right|=n^{-1 / 2} \max _{v \in T_{n}}\left|2 h_{L}(v)-h(v)\right| \stackrel{\mathrm{p}}{\longrightarrow} 0 .
$$

The result follows by Lemma 7.1 and (7.9).

Remark 7.4. It is known that the maxima in (7.9) actually are of the order $n^{1 / 4}$, see e.g. [33] and [13] for further results.

We return to permutations. Let $\pi \in \mathfrak{S}_{n}(132)$ be a 132-avoiding permutation and let $T$ be the correspondng binary tree defined in Section 4 . Label the vertices by the corresponding elements of $\pi$. (Thus the root is labelled by the maximum element $\pi_{\ell}=n$.) The inorder on $T$ corresponds to the standard order on the index set $[n]$; thus, the permutation $\pi$ can be recovered by taking the labels of $T$ in inorder. (This is why we need the inorder above.)

Define a partial order on the vertices of $T$ by $v \prec w$ if $v$ is an ancestor of $w$, i.e. lies on the path from the root to $w$. For two vertices $v$ and $w$, we let $v \wedge w$ be their last common ancestor (which is their greatest lower bound in this order). 
Let, as above, $v_{1}, \ldots, v_{n}$ be the vertices of $T$ in inorder; thus $v_{i}$ is labelled by $\pi_{i}$. Consider a pair of distinct $i, j \in[n]$. It follows from the construction of $T$ that if $v_{i} \prec v_{j}$, then $\pi_{i}>\pi_{j}$. Symmetrically, if $v_{j} \prec v_{i}$, then $\pi_{i}<\pi_{j}$. If neither holds, and $i<j$, then there exists a last common ancestor $v_{l}$ and then $i<l<j$ and $\pi_{l}>\pi_{i}>\pi_{j}$. Consequently, assuming $i<j$, we have

$$
\pi_{i}<\pi_{j} \Longleftrightarrow v_{j} \prec v_{i} .
$$

Theorem 7.5. Let $\sigma \in \mathfrak{S}_{k}(132)$ with $k \geqslant 1$. Then there exists a continuous functional $\Psi_{\sigma}$ on $C[0,1]$ such that $n^{-\lambda(\sigma) / 2} n_{\sigma}\left(\boldsymbol{\pi}_{132, n}\right) \stackrel{\mathrm{d}}{\longrightarrow} \Psi_{\sigma}(\mathbf{e})$ as $n \rightarrow \infty$; furthermore, $\Psi_{\sigma}(\mathbf{e})>0$ a.s.

Moreover, this holds jointly for all $\sigma$.

Proof. We say that an index $i \in[k]$ is black if either $i=1$ or $\sigma_{i-1}>\sigma_{i}$. (I.e., $i-1$ is 0 or a descent.) Otherwise, $i$ is white. Let $B$ be the set of black indices, and $W$ the set of white indices. Thus $|B|=D(\sigma)$ and $|W|=|\sigma|-D(\sigma)$.

Claim: If $i<j$ and $j$ is a black index, so $\sigma_{j-1}>\sigma_{j}$, then $\sigma_{i}>\sigma_{j}$, since otherwise $\sigma_{i} \sigma_{j-1} \sigma_{j}$ would be an occurence of 132 in $\sigma$.

Let $\nu_{1} \cdots \nu_{k}$ be a sequence with $1 \leqslant \nu_{1}<\cdots<\nu_{k} \leqslant n$ and let us investigate whether $\pi_{\nu_{1}} \cdots \pi_{\nu_{k}}$ is an occurrence of $\sigma$ in $\pi$. Write, for convenience, $\bar{v}_{i}=v_{\nu_{i}}$, the vertex in $T$ with label $\pi_{\nu_{i}}$. We say that $\nu_{i}$, or $\bar{v}_{i}$, is black or white if $i$ is.

We first consider $\nu_{i}$, or equivalently $\bar{v}_{i}$, for the black indices $i$. By the claim above, if $i$ and $j$ are black indices with $i<j$, then $\sigma_{i}>\sigma_{j}$ and thus we require $\pi_{\nu_{i}}>\pi_{\nu_{j}}$, which by (7.10) is equivalent to $\bar{v}_{j} \nprec \bar{v}_{i}$. The only condition for the black vertices $\bar{v}_{i}$ is thus that they are in increasing inorder and none is an ancestor of a previous one.

We then consider $\nu_{i}$ for the white indices, in order from left to right. For each white $j$ the conditions are as follows, by (7.10) and the claim above. Let $U_{j}:=\left\{i<j: \sigma_{i}<\sigma_{j}\right\}$ and note that $j-1 \in U_{j}$ since $j$ is white.

(i) $\nu_{j}>\nu_{i}$ for $i<j$.

(ii) $\bar{v}_{j} \prec \bar{v}_{i}$ for $i \in U_{j}$.

(iii) $\bar{v}_{j} \nprec \bar{v}_{i}$ for $i \in[j-1] \backslash U_{j}$.

(iv) $\nu_{j}<\nu_{i}$ and $\bar{v}_{i} \nprec \bar{v}_{j}$ for every black $i>j$.

Furthermore, let $b=b(j)$ be the largest black index in $[j-1]$.

The index $b \in U_{j}$ so by (ii)] $\bar{v}_{j} \prec \bar{v}_{b}$, i.e., $\bar{v}_{j}$ is on the path from the root to $\bar{v}_{b}$. Moreover, by (i), $\nu_{j}>\nu_{b}$, so $\bar{v}_{j}$ comes after $\bar{v}_{b}$ in the inorder; this means that the next step from $\bar{v}_{j}$ on the path to $\bar{v}_{b}$ is to the left. The number of such $\bar{v}_{j}$ (ignoring the other conditions) is $h_{L}\left(\bar{v}_{b}\right)$. For such $\bar{v}_{j}$, the condition (ii) that $\bar{v}_{j} \prec \bar{v}_{i}$ for $i \in U_{j}$ is equivalent to $\bar{v}_{j} \prec \bar{v}_{i} \wedge \bar{v}_{b}$, and thus to, if $i \leqslant b$,

$$
h_{L}\left(\nu_{j}\right)=h_{L}\left(\bar{v}_{j}\right)<h_{L}\left(\bar{v}_{i} \wedge \bar{v}_{b}\right)=h_{L}\left(\left[\nu_{i}, \nu_{b}\right]\right) ;
$$

if $i>b$, so also $\bar{v}_{i}$ is on the path to $\bar{v}_{b}$, the condition is simply

$$
h_{L}\left(\nu_{j}\right)<h_{L}\left(\nu_{i}\right)
$$


For $i \in[j-1] \backslash U_{j}$, which implies $i<b$, (iii) conversely requires

$$
h_{L}\left(\nu_{j}\right) \geqslant h_{L}\left(\left[\nu_{i}, \nu_{b}\right]\right) \text {. }
$$

In (iv), for black $i>j$, the condition $\bar{v}_{i} \nprec \bar{v}_{j}$ is redundant, since we already know $\bar{v}_{j} \prec \bar{v}_{b}$ and $\bar{v}_{i} \nprec \bar{v}_{b}$ (both $b$ and $i$ are black). Since $\bar{v}_{j} \prec \bar{v}_{b}$ and $\nu_{b}<\nu_{j}$, we see also that $\nu_{j}<\nu_{i}$ implies $\bar{v}_{j} \succeq \bar{v}_{b} \wedge \bar{v}_{i}$. (If $\bar{v}_{j} \prec \bar{v}_{b} \wedge \bar{v}_{i}$, then $\bar{v}_{b}$ and $\bar{v}_{i}$ are on the same side of $\bar{v}_{j}$.) This means

$$
h_{L}\left(\nu_{j}\right) \geqslant h_{L}\left(\left[\nu_{b}, \nu_{i}\right]\right) .
$$

Conversely, (7.11) -(7.14) are also sufficient for (i) (iv). (Note that (7.14) implies that $\bar{v}_{j} \in T_{L}\left(\bar{v}_{b} \wedge \bar{v}_{i}\right) \cup\left\{\bar{v}_{b} \wedge \bar{v}_{i}\right\}$ and $\bar{v}_{i} \in T_{R}\left(\bar{v}_{b} \wedge \bar{v}_{i}\right)$; thus $\nu_{j}<\nu_{i}$. Similarly, (i) follows from (17.11) -(7.13).)

Consequently, having chosen the black vertices, we have to choose $\nu_{j}$ for the white indices $j$ such that $\bar{v}_{j}$ is on the path from the root to $\bar{v}_{b(j)}$, with a left step next, and (7.11)-(7.14) hold.

Let us count. We choose first the black vertices, one by one. There are $\left(\begin{array}{c}n \\ D(\sigma)\end{array}\right)$ choices of $\left\{\nu_{i}: i \in B\right\}$. Of these, the condition $\bar{v}_{j} \nprec \bar{v}_{i}$ for $i<j$ forbids only $O(H)$ choices for each $j$ (where $H=H(T)$ is the height), and thus $O\left(n^{D(\sigma)-1} H\right)$ choices of the black vertices. We will simply ignore this restriction, introducing an error that will be negligible.

For each choice of black vertices, we then choose the white vertices $\bar{v}_{i}$. By the conditions above, there are at most $H$ choices for each white $\bar{v}_{i}$, and thus at most $H^{|W|}=H^{k-D(\sigma)}$ choices for $\left\{\bar{v}_{i}: i \in W\right\}$. More precisely, this number is a polynomial $\Phi=\Phi_{\sigma}$ of degree $k-D(\sigma)$ in the numbers $h_{L}\left(\nu_{i}\right)$ and $h_{L}\left(\left[\nu_{i}, \nu_{j}\right]\right), i, j \in B$. We will not attempt to give an exact description of this polynomial in general, but we give after the proof a few examples that will illustrate the construction, and it should be clear that similar constructions hold in general.

Let $B=\left\{b_{1}, \ldots, b_{D}\right\}$ where $D=D(\sigma)$. We regard $\Phi$ as a functional of the left profile $h_{L}$ and the black indices $\nu_{b_{1}}, \ldots, \nu_{b_{D}}$, and obtain

$$
n_{\sigma}(\pi)=\sum_{\nu_{b_{1}}<\cdots<\nu_{b_{D}}} \Phi\left(h_{L} ; \nu_{b_{1}}, \ldots, \nu_{b_{D}}\right)+O\left(n^{D-1} H \cdot H^{k-D}\right)
$$

where the error term comes from including also forbidden sets of black vertices.

We now use Lemma 7.3. By the Skorohod representation theorem, see Remark 7.2, we may assume that $\tilde{h}_{L}(x) \rightarrow 2^{1 / 2} \mathbf{e}(x)$ uniformly on $[0,1]$ as $n \rightarrow \infty$. In particular this implies that $n^{-1 / 2} \max _{i} h_{L}(i)=\sup _{x \in[0,1]} \tilde{h}_{L}(x)=$ $O(1)$. (The implicit constant is random but does not depend on $n$.) Similarly, by Lemma 7.1, we may assume $n^{-1 / 2} H=n^{-1 / 2} \max _{i} h(i)=O(1)$, i.e., $H=O\left(n^{1 / 2}\right)$.

Letting $\Phi^{\prime}$ be the leading terms in $\Phi$, which are homogeneous of degree $|W|=k-D$, and letting $\widetilde{\Phi}$ be the corresponding functional for functions 
on $[0,1]$, we then obtain from (7.15),

$$
\begin{aligned}
n_{\sigma}\left(\boldsymbol{\pi}_{132, n}\right)= & \sum_{\nu_{b_{1}}<\cdots<\nu_{b_{D}}} \Phi^{\prime}\left(h_{L} ; \nu_{b_{1}}, \ldots, \nu_{b_{D}}\right)+ \\
& +O\left(n^{D} H^{k-D-1}\right) \\
= & n^{(k-D) / 2} \sum_{0 \leqslant i_{1}<\cdots<i_{D} \leqslant n-1} \widetilde{\Phi}\left(\tilde{h}_{L} ; i_{1} / n, \ldots, i_{D} / n\right)+O\left(n^{(D+k-1) / 2}\right) \\
= & n^{(k+D) / 2} \int_{0 \leqslant x_{1}<\cdots<x_{D} \leqslant 1} \widetilde{\Phi}\left(\tilde{h}_{L} ; x_{1}, \ldots, x_{D}\right) \mathrm{d} x_{1} \cdots \mathrm{d} x_{D} \\
& +O\left(n^{(D+k-1) / 2}\right) .
\end{aligned}
$$

We define, for a function $f$ on $[0,1]$,

$$
\Psi_{\sigma}(f):=2^{(k-D) / 2} \int_{0 \leqslant x_{1}<\cdots<x_{D} \leqslant 1} \widetilde{\Phi}\left(f ; x_{1}, \ldots, x_{D}\right) \mathrm{d} x_{1} \cdots \mathrm{d} x_{D}
$$

and have thus, by the uniform convergence $\tilde{h}_{L} \rightarrow 2^{1 / 2} \mathbf{e}$,

$$
n^{-(k+D) / 2} n_{\sigma}\left(\boldsymbol{\pi}_{132, n}\right)=\Psi_{\sigma}\left(2^{-1 / 2} \tilde{h}_{L}\right)+o(1) \rightarrow \Psi_{\sigma}(\mathbf{e}) .
$$

It is obvious that $\Psi_{\sigma}(\mathbf{e})>0$ a.s. This completes the proof.

Example 7.6. $\sigma=12 . B=\{1\}$. For every choice of the black $\nu_{1}$, there are $h_{L}\left(\nu_{1}\right)$ choices of the white $\nu_{2}$. Hence (7.15) is simply $n_{\sigma}(\pi)=\sum_{\nu=1}^{n} h_{L}(\nu)$ and $\Phi\left(h_{L} ; \nu\right)=h_{L}(\nu)$. Consequently $\widetilde{\Phi}(f ; x)=f(x)$ and (7.16) yields

$$
\Lambda_{12}=X_{12}(T)=\Psi_{12}(\mathbf{e})=\sqrt{2} \int_{0}^{1} \mathbf{e}(x) \mathrm{d} x .
$$

As said in Section 2, this is, apart from the factor $\sqrt{2}$, the well-known Brownian excursion area.

Example 7.7. $\sigma=123$. $B=\{1\}$. Both $\bar{v}_{2}$ and $\bar{v}_{3}$ are on the path to $\bar{v}_{1}$, both with the next step left, and with $\bar{v}_{3} \prec \bar{v}_{2}$. There are $\left(\begin{array}{c}h_{L}\left(\nu_{1}\right) \\ 2\end{array}\right)$ choices of them for each $\nu_{1}$. Hence,

$$
n_{123}(\pi)=X_{123}(T)=\sum_{\nu=1}^{n}\left(\begin{array}{c}
h_{L}(\nu) \\
2
\end{array}\right)
$$

which leads to $\widetilde{\Phi}(f ; x)=\frac{1}{2} f(x)^{2}$ and

$$
\Lambda_{123}=\Psi_{123}(\mathbf{e})=\int_{0}^{1} \mathbf{e}(x)^{2} \mathrm{~d} x .
$$

The joint distribution of the random variables $\int_{0}^{1} \mathbf{e}$ (see Example [7.6) and $\int_{0}^{1} \mathbf{e}^{2}$ have been studied by Nguyen The [35], who found a recursion for mixed moments equivalent to Theorem 6.5](i). He also found the Laplace transform $\mathbb{E} e^{-t \Lambda_{123}}=(\sqrt{2 t} / \sinh (\sqrt{2 t}))^{3 / 2}$, which shows that $\Lambda_{123}$ has the distribution denoted $S_{3 / 2}$ in Biane, Pitman and Yor [5], see in particular [5, 
Secion 4.4] (and recall that e can be seen as a 3-dimensional Bessel bridge). Equivalently, $\Lambda_{123}$ has the moment generating function

$$
\mathbb{E} e^{t \Lambda_{123}}=\left(\frac{\sqrt{2 t}}{\sin (\sqrt{2 t})}\right)^{3 / 2}, \quad \operatorname{Re} t<\frac{\pi^{2}}{2} .
$$

Example 7.8. More generally, for $\sigma=1 \cdots k$, any $k \geqslant 1$,

$$
n_{1 \cdots k}(\pi)=X_{1 \cdots k}(T)=\sum_{\nu=1}^{n}\left(\begin{array}{c}
h_{L}(\nu) \\
k-1
\end{array}\right)
$$

and

$$
\Lambda_{1 \cdots k}=\Psi_{1 \cdots k}(\mathbf{e})=\frac{2^{(k-1) / 2}}{(k-1) !} \int_{0}^{1} \mathbf{e}(x)^{k-1} \mathrm{~d} x .
$$

Thus, if $Z_{k}:=\int_{0}^{1} \mathbf{e}(x)^{k}$, the average of the $k:$ th power of the Brownian excursion, then $\Lambda_{1 \cdots k}=c_{k} Z_{k-1}$ with $c_{k}=2^{(k-1) / 2} /(k-1)$ !. The random variables $Z_{k}$ have been studied by Richard [37]; in particular, [37] gives a recursion formula for the mixed moments, which is equivalent to our recursion implicit in the proof of Lemma 6.1 for this case.

Note that, by Hölder's inequality, $Z_{k} \geqslant Z_{1}^{k}$ for every $k$; hence by the known asymptotics for moments of the Brownian excursion area $Z_{1}$, see e.g. [24], as $r \rightarrow \infty$,

$$
\mathbb{E} Z_{k}^{r} \geqslant \mathbb{E} Z_{1}^{k r} \sim 3 \sqrt{2} k r\left(\frac{k r}{12 e}\right)^{k r / 2} .
$$

More precisely, it follows from [19, Theorem 2.1] (applied to $Z_{k}^{1 / k}$ ) that for every fixed $k \geqslant 1$, as $r \rightarrow \infty$,

$$
\left(\mathbb{E} Z_{k}^{r}\right)^{1 / r} \sim z_{k} r^{k / 2}
$$

where $z_{k}>0$ is a constant given by

$$
z_{k}:=\left(\frac{k}{e}\right)^{k / 2} \max \left\{\int_{0}^{1} f(x)^{k}: f(0)=f(1)=0 \text { and } \int_{0}^{1}\left(f^{\prime}(x)\right)^{2} \leqslant 1\right\} .
$$

(We have $z_{1}=1 / \sqrt{12 e}$ and $z_{2}=2 /\left(e \pi^{2}\right)$; we do not know $z_{k}$ for $k>2$.)

It follows from (7.24) $-(7.25)$ that the moment generating function $\mathbb{E} e^{t Z_{k}}$ of $Z_{k}$ is an entire function for $k=1$ (see further [24]), but has a finite radius of convergence for $k=2$ and diverges for all $t>0$ when $k \geqslant 3$. (The claim in [37, Theorem 1.2] that $Z_{1}, \ldots, Z_{M}$ have an entire moment generating function is thus incorrect. For $Z_{2}$ this is also seen by the explicit formula (7.21).)

Moreover, the Carleman condition (in its weaker form for nonnegative random variables, see e.g. [21, Section 4.10])

$$
\sum_{m}\left(\mathbb{E} Z_{k}^{m}\right)^{-1 / 2 m}=\infty
$$

holds by (7.25) for $k \leqslant 4$ but not for $k \geqslant 5$. Although the Carleman condition is only sufficient for a distribution to be determined by its moments, 
this strongly suggests that the distribution of $Z_{k}$, and thus $\Lambda_{1 \ldots(k+1)}$, is not determined by its moments if $k$ is large enough.

Example 7.9. $\sigma=213 . \quad B=\{1,2\}$. Given $\bar{v}_{1}$ and $\bar{v}_{2}$, the white vertex $\bar{v}_{3}$ has to be on the path to $\bar{v}_{1} \wedge \bar{v}_{2}$. There are $h_{L}\left(\bar{v}_{1} \wedge \bar{v}_{2}\right)=h_{L}\left(\left[\nu_{1}, \nu_{2}\right]\right)$ choices, and thus

$$
n_{213}(\pi)=X_{213}(T)=\sum_{\nu_{1}<\nu_{2}} h_{L}\left(\left[\nu_{1}, \nu_{2}\right]\right)+O\left(n H^{2}\right)
$$

which leads to

$$
\Lambda_{213}=\Psi_{213}(\mathbf{e})=\sqrt{2} \iint_{0 \leqslant x<y \leqslant 1} \mathbf{e}([x, y]) \mathrm{d} x \mathrm{~d} y .
$$

Example 7.10. $\sigma=231$. $B=\{1,3\}$. Given $\bar{v}_{1}$ and $\bar{v}_{3}$, the white vertex $\bar{v}_{2}$ has to be on the path to $\bar{v}_{1}$ but not to $\bar{v}_{3}$. There are $h_{L}\left(\bar{v}_{1}\right)-h_{L}\left(\bar{v}_{1} \wedge \bar{v}_{3}\right)-1$ choices, and thus

$$
n_{231}(\pi)=X_{231}(T)=\sum_{\nu_{1}<\nu_{3}}\left(h_{L}\left(\bar{v}_{1}\right)-h_{L}\left(\left[\nu_{1}, \nu_{3}\right]\right)-1\right)+O\left(n H^{2}\right)
$$

which leads to

$$
\Lambda_{231}=\Psi_{231}(\mathbf{e})=\sqrt{2} \iint_{0 \leqslant x<y \leqslant 1}(\mathbf{e}(x)-\mathbf{e}([x, y])) \mathrm{d} x \mathrm{~d} y .
$$

Example 7.11. $\sigma=312 . B=\{1,2\}$. Given $\bar{v}_{1}$ and $\bar{v}_{2}$, the white vertex $\bar{v}_{3}$ has to be on the path to $\bar{v}_{2}$ but not to $\bar{v}_{1}$. Thus

$$
n_{312}(\pi)=X_{312}(T)=\sum_{\nu_{1}<\nu_{2}}\left(h_{L}\left(\bar{v}_{2}\right)-h_{L}\left(\left[\nu_{1}, \nu_{2}\right]\right)-1\right)+O\left(n H^{2}\right)
$$

which leads to

$$
\Lambda_{312}=\Psi_{312}(\mathbf{e})=\sqrt{2} \iint_{0 \leqslant x<y \leqslant 1}(\mathbf{e}(y)-\mathbf{e}([x, y])) \mathrm{d} x \mathrm{~d} y .
$$

Note that the equality in distribution $\Lambda_{231} \stackrel{\mathrm{d}}{=} \Lambda_{312}$ here is immediate by (7.29), (7.31) and the symmetry $\mathbf{e}(x) \stackrel{\mathrm{d}}{=} \mathbf{e}(1-x)$ of the Brownian excursion. However, we see also that $\Lambda_{231}$ and $\Lambda_{312}$ differ as random variables, which means that the joint distribution of $n_{231}$ and $n_{312}$ does not have degenerate (one-dimensional) asymptotic distribution. Cf. the second moments in (6.19).

Furthermore, note that the identity (2.14) also can be seen from (17.18), (17.27), (7.29), (7.31).

Remark 7.12. Janson [23] studied some functionals of random trees and found as limits in distribution three functionals of Browninan excursion, there denoted $\xi, \eta, \zeta . \xi$ is simply twice the Brownian excursion area, so by (7.18), $\xi=\sqrt{2} \Lambda_{12}$. Furthermore, $\eta$ is 4 times the integral in (17.27), and thus $\eta=2^{3 / 2} \Lambda_{213}$. Finally, $\zeta=\xi-\eta=2^{1 / 2}\left(\Lambda_{231}+\Lambda_{312}\right)$, by (2.14) or by comparing the formula in [23] to (7.29) and (7.31). 
Example 7.13. $\sigma=k \cdots 1$. This is the trivial case when all vertices are black, so $\Phi=1$ is constant. Thus also $\widetilde{\Phi}=1$ and (7.16) yields $\Psi_{k \cdots 1}=1 / k$ !, in accordance with Theorem 2.1 and Remark 2.4.

Although the expressions get increasingly more complicated, it is clear that there are of the same nature for every $\sigma$. In particular, except for the case $\sigma=k \cdots 1$, see Example 7.13, $\Psi_{\sigma}(\mathbf{e})$ is non-degenerate, i.e., not a.s. constant.

\section{Proof of Theorem 2.1}

Theorem 7.5 shows the existence of limits $\Lambda_{\sigma}=\Psi_{\sigma}(\mathbf{e})$ such that (2.3) holds, jointly for all $\sigma \in \mathfrak{S}_{*}(132)$. Furthermore, $\Lambda_{\sigma}>0$ a.s. and $\Lambda_{\sigma}$ is non-degenerate except in the case $\sigma=k \cdots 1$.

On the other hand, Lemmas 6.1 and 5.4 show that for any $\sigma^{(1)}, \ldots, \sigma^{(M)}$,

$$
n^{-\sum_{\nu} \lambda\left(\sigma^{(\nu)}\right) / 2} \mathbb{E}\left(n_{\sigma^{(1)}} \cdots n_{\sigma^{(M)}}\left(\boldsymbol{\pi}_{132, n}\right)\right) \rightarrow A_{\sigma^{(1)}, \ldots, \sigma^{(M)}}
$$

for some constant $A_{\sigma^{(1)}, \ldots, \sigma^{(M)}}<\infty$. As is well-known, convergence of all moments implies that all products $n_{\sigma^{(1)}} \cdots n_{\sigma^{(M)}}\left(\boldsymbol{\pi}_{132, n}\right)$ are uniformly integrable, and thus the limits of the moments are the moments of the limits $\Lambda_{\sigma} \cdot$ [21, Theorems 5.4.2 and 5.5.9].

Remark 8.1. Note that we cannot use (8.1) to show the existence of limits $\Lambda_{\sigma}$ in (2.3), since we cannot show that the limit distributions are determined by thier moments; on the contrary, we believe that they in general are not, see Example 7.8. This is one reason for using two different methods in the proof above, one for the existence of limits in distribution and another for the limits of moments.

\section{Further COMMENTS}

As said in Remark 2.3, Bóna [10] has shown that for every $n$ and any $\sigma \in \mathfrak{S}_{k}(132)$,

$$
\mathbb{E} n_{1 \cdots k}\left(\boldsymbol{\pi}_{132, n}\right) \leqslant \mathbb{E} n_{\sigma}\left(\boldsymbol{\pi}_{132, n}\right) \leqslant \mathbb{E} n_{k \cdots 1}\left(\boldsymbol{\pi}_{132, n}\right) .
$$

We use here the recursion Lemma 3.2 to show a more general result.

Define a partial order on each $\mathfrak{S}_{k}(132)$ by

$$
\sigma \prec \sigma^{\prime} \Longleftrightarrow\left\{(i, j): i<j \text { and } \sigma_{i}>\sigma_{j}\right\} \subseteq\left\{(i, j): i<j \text { and } \sigma_{i}^{\prime}>\sigma_{j}^{\prime}\right\} \text {. }
$$

Theorem 9.1. If $|\sigma|=\left|\sigma^{\prime}\right|$ and $\sigma \prec \sigma^{\prime}$, then $\mathbb{E} n_{\sigma}\left(\boldsymbol{\pi}_{132, n}\right) \leqslant \mathbb{E} n_{\sigma^{\prime}}\left(\boldsymbol{\pi}_{132, n}\right)$ for every $n \geqslant 1$.

Note that $1 \cdots k$ is minimal and $k \cdots 1$ is maximal in the partial order $\prec$, so (9.1) follows immediately.

Proof. We use induction on $n$. The case $n=1$ is trivial.

Condition on the value of the maximal index $\ell$ in $\pi=\pi_{132, n}$. Given $\ell, \pi_{L}$ and $\pi_{R}$ are independent uniformly random elements of $\mathfrak{S}_{\ell-1}(132)$ 
and $\mathfrak{S}_{n-\ell}(132)$ respectively. Furthermore, $\sigma \prec \sigma^{\prime}$ implies that $\sigma_{1} \cdots \sigma_{q} \prec$ $\sigma_{1}^{\prime} \cdots \sigma_{q}^{\prime}$ and $\sigma_{q+1} \cdots \sigma_{k} \prec \sigma_{q+1}^{\prime} \cdots \sigma_{k}^{\prime}$ for every $q \in[k]$, and also $\Delta_{\sigma} \subseteq \Delta_{\sigma^{\prime}}$.

Using (3.1) for both $\sigma$ and $\sigma^{\prime}$ and taking the conditional expectations, it follows, by this and the induction hypothesis, that

$$
\mathbb{E}\left(n_{\sigma}\left(\boldsymbol{\pi}_{132, n}\right) \mid \ell\right) \leqslant \mathbb{E}\left(n_{\sigma^{\prime}}\left(\boldsymbol{\pi}_{132, n}\right) \mid \ell\right)
$$

for every value of $\ell \in[n]$. Taking the expectation we obtain $\mathbb{E} n_{\sigma}\left(\boldsymbol{\pi}_{132, n}\right) \leqslant$ $\mathbb{E} n_{\sigma^{\prime}}\left(\boldsymbol{\pi}_{132, n}\right)$, completing the induction step.

Rudolph [39] has a general result, and a conjecture, for the related problem of when there is equality $\mathbb{E} n_{\sigma}\left(\boldsymbol{\pi}_{132, n}\right)=\mathbb{E} n_{\sigma^{\prime}}\left(\boldsymbol{\pi}_{132, n}\right)$ for all $n$. It seems possible that Lemma 3.2 can be used to prove, and perhaps improve, her results too, but we have not attempted this.

\section{REFERENCES}

[1] M. H. Albert, M. D. Atkinson and Robert Brignall, The enumeration of permutations avoiding 2143 and 4231. Pure Math. Appl. 22 (2011), no. 2, 87-98.

[2] M. H. Albert, M. D. Atkinson and Robert Brignall, The enumeration of three pattern classes using monotone grid classes. Electron. J. Combin. 19 (2012), no. 3, Paper 20, 34 pp.

[3] David Aldous, The continuum random tree II: an overview. Stochastic Analysis (Durham, 1990), 23-70, London Math. Soc. Lecture Note Ser. 167, Cambridge Univ. Press, Cambridge, 1991.

[4] David Aldous, The continuum random tree III. Ann. Probab. 21 (1993), no. 1, 248-289.

[5] Philippe Biane, Jim Pitman and Marc Yor, Probability laws related to the Jacobi theta and Riemann zeta functions, and Brownian excursions. Bull. Amer. Math. Soc. (N.S.) 38 (2001), no. 4, 435-465.

[6] Sara C. Billey, William Jockusch and Richard P. Stanley, Some combinatorial properties of Schubert polynomials. J. Algebraic Combin. 2 (1993), no. 4, 345-374.

[7] Patrick Billingsley, Convergence of Probability Measures. Wiley, New York, 1968.

[8] Miklós Bóna, Combinatorics of Permutations. Chapman \& Hall/CRC, Boca Raton, FL, 2004.

[9] Miklós Bóna, The copies of any permutation pattern are asymptotically normal. Preprint, 2007. arXiv:0712.2792.

[10] Miklós Bóna, The absence of a pattern and the occurrences of another. Discrete Math. Theor. Comput. Sci. 12 (2010), no. 2, 89-102.

[11] Miklós Bóna, On three different notions of monotone subsequences. Permutation Patterns, 89-114, London Math. Soc. Lecture Note Ser., 376, Cambridge Univ. Press, Cambridge, 2010.

[12] Miklós Bóna, Surprising symmetries in objects counted by Catalan numbers. Electron. J. Combin. 19 (2012), no. 1, Paper 62, 11 pp. 
[13] Mireille Bousquet-Mélou and Svante Janson, The density of the ISE and local limit laws for embedded trees. Ann. Appl. Probab. 16 (2006), no. 3, 1597-1632.

[14] Szu-En Cheng, Sen-Peng Eu and Tung-Shan Fu, Area of Catalan paths on a checkerboard. European J. Combin. 28 (2007), no. 4, 1331-1344.

[15] Timothy Chow and Julian West, Forbidden subsequences and Chebyshev polynomials. Discrete Math. 204 (1999), no. 1-3, 119-128.

[16] Joshua Cooper, Combinatorial problems I like. http://www.math.sc.edu/ cooper/combprob.html

[17] Michael Drmota, Random Trees. Springer, Vienna, 2009.

[18] Richard T. Durrett, Donald L. Iglehart and Douglas R. Miller, Weak convergence to Brownian meander and Brownian excursion. Ann. Probability 5 (1977), no. 1, 117-129.

[19] James Allen Fill and Svante Janson, Precise logarithmic asymptotics for the right tails of some limit random variables for random trees. Ann. Comb. 12 (2009), no. 4, 403-416.

[20] Philippe Flajolet and Robert Sedgewick, Analytic Combinatorics. Cambridge Univ. Press, Cambridge, UK, 2009.

[21] A. Gut, Probability: A Graduate Course. 2nd ed, Springer, New York, 2013.

[22] Cheyne Homberger, Expected patterns in permutation classes. Electron. J. Combin. 19 (2012), no. 3, Paper 43, 12 pp.

[23] Svante Janson, The Wiener index of simply generated random trees. Random Structures Algorithms 22 (2003), no. 4, 337-358.

[24] Svante Janson, Brownian excursion area, Wright's constants in graph enumeration, and other Brownian areas. Probab. Surv. 4 (2007), 80-145.

[25] Svante Janson, Brian Nakamura and Doron Zeilberger, On the asymptotic statistics of the number of occurrences of multiple permutation patterns. Preprint, 2013. arXiv:1312.3955.

[26] Olav Kallenberg, Foundations of Modern Probability. 2nd ed., Springer, New York, 2002.

[27] Donald E. Knuth, The Art of Computer Programming. Vol. 1: Fundamental Algorithms. 3nd ed., Addison-Wesley, Reading, Mass., 1997.

[28] C. Krattenthaler, Permutations with restricted patterns and Dyck paths. Adv. in Appl. Math. 27 (2001), no. 2-3, 510-530.

[29] G. Louchard, The Brownian excursion area: a numerical analysis. Comput. Math. Appl. 10 (1984), no. 6, 413-417. Erratum: Comput. Math. Appl. Part A 12 (1986), no. 3, 375.

[30] Toufik Mansour and Alek Vainshtein, Restricted permutations, continued fractions, and Chebyshev polynomials. Electron. J. Combin. 7 (2000), Research Paper 17, 9 pp.

[31] Toufik Mansour and Alek Vainshtein, Restricted 132-avoiding permutations. Adv. in Appl. Math. 26 (2001), no. 3, 258-269.

[32] T. Mansour and A. Vainshtein, Restricted permutations and Chebyshev polynomials. Sém. Lothar. Combin. 47 (2001/02), Article B47c, 17 pp. 
[33] Jean-François Marckert, The rotation correspondence is asymptotically a dilatation. Random Structures Algorithms 24 (2004), no. 2, 118-132.

[34] Jean-François Marckert and Abdelkader Mokkadem, The depth first processes of Galton-Watson trees converge to the same Brownian excursion. Ann. Probab. 31 (2003), no. 3, 1655-1678.

[35] Michel Nguyen The, Area and inertial moment of Dyck paths. Combin. Probab. Comput. 13 (2004), no. 4-5, 697-716.

[36] Daniel Revuz and Marc Yor, Continuous Martingales and Brownian Motion. $3^{\text {rd }}$ edition, Springer-Verlag, Berlin, 1999.

[37] Christoph Richard, On $q$-functional equations and excursion moments. Discrete Math. 309 (2009), no. 1, 207-230.

[38] Aaron Robertson, Herbert S. Wilf and Doron Zeilberger, Permutation patterns and continued fractions. Electron. J. Combin. 6 (1999), Research Paper 38, 6 pp.

[39] Kate Rudolph, Pattern popularity in 132-avoiding permutations. Electron. J. Combin. 20 (2013), no. 1, Paper 8, 15 pp.

[40] Rodica Simion and Frank W. Schmidt, Restricted permutations. European J. Combin. 6 (1985), no. 4, 383-406.

[41] Richard P. Stanley, Enumerative Combinatorics, Volume 2, Cambridge Univ. Press, Cambridge, 1999.

[42] Julian West, Generating trees and forbidden subsequences. Discrete Math. 157 (1996), no. 1-3, 363-374.

Department of Mathematics, Uppsala University, PO Box 480, SE-751 06 UPPSAla, SWEDEN

E-mail address: svante.janson@math.uu.se

$U R L:$ http://www2.math.uu.se/ svante/ 